This document is the accepted manuscript version of the following article:

Authors: Alice Berardo, Nicola M. Pugno

Title: A model for hierarchical anisotropic friction, adhesion and wear

Journal: Tribology International

Year: 2020

Accepted date: 5 July 2020

DOI: $10.1016 /$ j.triboint.2020.106549

This manuscript version is made available under the CC-BY-NC-ND 4.0 license

Originally uploaded to URL: http://www.ing.unitn.it/ pugno/NP_PDF/PostPrint/2020Berardo-model.pdf on /20/09/2020 
CRediT author statement

A.B. Conceptualization, Methodology, Software; Validation, Formal analysis, Investigation, Data curation, Writing - original draft; Writing - review and editing, Visualization.

N.M.P. Conceptualization, Methodology, Supervision, Writing - review and editing, Project administration, Funding acquisition. 


\title{
A model for hierarchical anisotropic friction, adhesion and wear
}

\author{
Alice Berardo ${ }^{1,2}$, Nicola M. Pugno ${ }^{1,3^{*}}$ \\ ${ }^{1}$ Laboratory of Bio-inspired, Bionic, Nano, Meta Materials \& Mechanics, Department of Civil, Environmental and \\ Mechanical Engineering, University of Trento, Via Mesiano 77, 38123 Trento, Italy \\ ${ }^{2}$ Fondazione Edmund Mach, Via Edmund Mach, 1 - 38010 San Michele all'Adige (TN), Italy \\ ${ }^{3}$ School of Engineering and Materials Science, Queen Mary University of London, Mile End Road, London E1 4NS, \\ U.K.
}

*Corresponding author: nicola.pugno@unitn.it; Tel.: +390461282525

\begin{abstract}
.
Due to a widespread number of examples, biological hierarchical structures have been studied for years, founding that surface properties are strictly correlated to the surface roughness. Since friction appears to be a multiscale phenomenon and hierarchy optimizes the macroscopic mechanical properties of bodies, it may be of great interest to develop a model to predict friction of structures with hierarchical surfaces.

Thus, we propose an analytical model to describe anisotropic friction, adhesion and wear of hierarchical surfaces. The model describes friction between two generic rough surfaces in contact, sliding one against the other. Then, it has been extended to adhesion, wear and finally to multiple hierarchical levels to obtain the global frictional response.
\end{abstract}

Keywords. Anisotropic friction, hierarchy, roughness, adhesion.

\section{Introduction}

Although it has been studied for centuries, friction still includes various open questions due to the many aspects occurring at the interface between bodies in relative motion.

Leonardo da Vinci first proposed the "laws of dry friction", then reformulated and collected by Amontons in the so-called four "laws of friction", which are the basic qualitative concepts related to this very complex phenomenon [1,2].

Despite their simplicity, these laws resulted in the Amontons-Coulomb constitutive laws of friction [3-5], summarized as follows:

- The friction resistance increases or decreases proportionally to the applied pressure;

- The friction coefficient (which is the ratio between the friction force and the normal load) is independent of the sliding velocity, contact area and surface roughness [2,4-6].

Coulomb stated that "Friction and cohesion are not active forces like gravity, but only passive forces" [2], highlighting that they arise only in the presence of an incipient relative motion (static friction force) or during the sliding (dynamic friction force). He also generalized the relationship between the tangential force and the applied load, by introducing a cohesive contribution, independent of the normal pressure.

The frictional stress $\tau$ is expressed as follows:

$$
\tau=\tau_{0}+\mu \sigma_{N}
$$

where $\tau_{0}$ is the cohesive component, $\sigma_{N}$ is the normal pressure and $\mu$ is the internal coefficient of friction.

The Coulomb's law is still considered the basis for the description of dry friction in classical mechanics, even though recent studies have proved that some deviations may occur [7-10]. For example, it does not consider the presence of anisotropy, i.e. when the friction force depends on the direction of sliding, or how the normal load depends on the real contact area.

Over the years, analytical models have been introduced to study the complicated problem of friction. One of the main contact theories was introduced by Hertz [11], which paved the way for the development of more 
recent models for contact mechanics, such as the one proposed by Greenwood and Williamson (GW) [12], the so called multi-asperity model and the related studies by Majumdar and Bhusham $[13,14]$, or alternative solutions, such as Persson's theory $[3,15,16]$. They presented different approaches to compute the response of a rough surface in contact with a flat substrate, due to a normal load. Greenwood and Williamson developed the Archard's studies, assuming a surface formed by $n$ elastic spherical caps with equal radii but different heights and provided the solution for the contact area after pressing this multi-asperity surface against a rigid plane $[12,17,18]$. They found that the area of real contact, usually smaller than the apparent contact area [6], is proportional to the load, while the average size of micro contacts is independent of the load. Indeed, when the load increases, new contact spots are generated. On the contrary, Persson referred to surface roughness by defining the power spectral density of the undeformed rough surface and studied the contact mechanics between this rough rigid surface and a semi-infinite elastic solid.

Other models have been developed during the years to study the interaction between the asperities of rough surfaces. The one proposed by Caroli and Nozières [19] studied velocity independent friction in a dry system and found that it is generated by hysteretic behaviour, while the elastic interactions between the asperities play a minor role in solid friction of multicontact interfaces. Mroz and Stupkiewicz [20] reported a friction model for anisotropic friction, based on the local validity of the classical Coulomb's law [6], which considers parallel springs to simulate the interaction between rough surfaces and that was also applied more recently in [21].

However, friction is not only a macroscopic problem, but it also involves several effects that occur at the micro and nanoscale, from adhesion to asperity interactions [22,23], which can also influence the mechanical properties of materials e.g. toughness for microfibers [24,25].

As reported in past and recent works [7-10,20,26-28], contact between rough surfaces is an example of a multiscale problem, sometimes leading to hierarchical and fractal geometries [29-33]. Indeed, biological structures have efficient ways to minimize or maximize friction or to adapt to different environments depending on the goals $[34,35]$. Usually, these intriguing properties have been developed thanks to a specific hierarchical structure. By changing their organization, variations in the mechanical and physical properties are possible. For this reason, systems and structures in nature draw a lot of attention thanks to their high efficiency and durability [36-38] and to date, the interest in transferring technologies from biological systems into engineering applications has been greatly pursued in the field of tribology.

This being the case, in the proposed paper an analytical model for anisotropic dynamic friction is introduced, which directly connects roughness with the friction coefficient. The model has been extended from [20] by introducing one-dimensional (1D) and two-dimensional (2D) roughness. Subsequently, other aspects have been developed as (i) adhesion, modelled as a tensile force increasing its effects during surfaces separation, (ii) wear, introduced to modify the roughness profile, according to Archard's law [18], and (iii) hierarchy, studying the contribution of fractal surfaces to the frictional response of the system $[23,39]$.

\section{The Anisotropic Lattice Spring Friction Model (ALSFM)}

Suppose to have two rough surfaces in contact, modelled as reported in Figure 1.a, where roughness can be expressed by a continuous and differentiable function with a defined periodicity in the form $z=f(x, y)$, here called 2D roughness (Figure 1.b).

Thus, these surfaces are:

$$
\begin{aligned}
& z_{1}=f_{1}(x, y) \\
& z_{2}=f_{2}(x, y)
\end{aligned}
$$

where the lower surface is identified by no. 1 , while the upper is no. 2 .

The analytical model assumes that the isotropic Coulomb friction law governs the local contact between the rough surfaces, already in relative motion, thus it considers only the dynamic friction force. These surfaces are assumed to be infinitely rigid, while a set of longitudinal springs, equally spaced and with the same stiffness, governs their elastic interaction (Figure 1.a). 
It has been supposed that the springs are fixed half on the upper surface and slide on the lower, and the other half opposite to them, to consider the contributions of both the rough surfaces to the global coefficient of friction.

As a first approach we neglect adhesion, defining that the springs work only when compressed with the same longitudinal stiffness $k_{c}$.

Firstly, we analyse the force equilibrium at point $P(P \in z(x, y))$ due to a single spring interaction (Figure 1.b-c) on the plane $\pi$, tangent to the surface in $P$ :

$$
\begin{gathered}
\left(x-x_{P}\right) \frac{\partial G}{\partial x}+\left(y-y_{P}\right) \frac{\partial G}{\partial y}+\left(z-z_{P}\right) \frac{\partial G}{\partial z}=0 \\
G: f(x, y)-z=0
\end{gathered}
$$

Assume the spring is fixed on the upper asperity (surface $z_{2}$ ) and slides on the lower (surface $z_{1}$ ); $l_{0}$ is the spring length at rest: the actual spring length $l$ depends on the asperities profile, thus, for point $P$, its length is equal to:

$$
l_{p}=z_{2}\left(x_{p}, y_{p}\right)-z_{1}\left(x_{p}, y_{p}\right)
$$

Due to its length variation during the sliding, the single spring generates an elastic force, expressed as follows:

$$
\begin{gathered}
F_{Z}(P)=\left(l_{0}-l_{P}\right) k_{c} \text { in case of compression; } \\
F_{z}(P)=0 \text { in case of elongation, since the tensile behaviour is neglected; }
\end{gathered}
$$

From the static equilibrium, $N$ is the normal force acting on the $\pi$ plane (Figure 1.c) and the tangential force is equal to $T=\mu_{0} N$, where $\mu_{0}$ is the local coefficient of friction (following the isotropic Coulomb law).

Supposing that the spring displacement $\left(l_{0}-l_{P}\right)$ is $u$, the normal force on $\pi$ is:

$$
N=k u\left[\cos \varphi_{P}-\frac{\mu_{0} \tan \varphi_{P} \cos \beta}{\sqrt{1+\tan ^{2} \varphi_{P} \cos ^{2} \beta}}\right]^{-1}
$$

$\beta$ is the projected direction on the $x^{\prime} y^{\prime}$ plane of the sliding velocity vector $v_{0}$ acting on the $\pi$ plane, tilted by $\varphi_{P}$ from $x^{\prime} y^{\prime}$ plane, $\varphi_{P}$ is the intersection between the tangent plane in $P$ and the $x^{\prime} y^{\prime}$ plane, as reported in Figure 1.c.

The denominator in Eq. (1) should be different from zero, leading to have $\varphi_{P} \neq 90^{\circ}$ and $\mu_{0} \neq \cot \varphi_{P}$ $\forall P \in z(x, y)$. When $F_{z}$ is a compressive force, $k u \geq 0$ and $\cos \varphi_{P}-\frac{\mu_{0} \tan \varphi_{P} \cos \beta}{\sqrt{1+\tan ^{2} \varphi_{P} \cos ^{2} \beta}}>0$. This means that $\left(l_{0}-l_{P}\right)$ is positive and that the local coefficient of friction $\mu_{0}$ must be smaller than $\cot \varphi_{P}$.

This means the more the material displays sharp roughness, the more the local friction coefficient must be small and in particular, for $\varphi$ equal to $45^{\circ}, \mu_{0}$ has to be minor than 1. From a physical point of view, Eq. (1) implies that the model is suitable for elastomers only when they show a smooth roughness, since they usually display dynamic coefficient of friction around 1-2 [40-43]. In addition, in case of sharp-shaped asperities $(\varphi$ $=60^{\circ}$ or more), the global coefficient of friction $\mu$ strongly increases and for $\varphi=90^{\circ}$ tends to an infinite value. This condition can model the presence of interlocking when the asperities are sharp, since the friction force significantly grows, affecting the sliding and even stopping it. In the $x^{\prime} y^{\prime}$ plane, from the equilibrium, the tangential forces are expressed in terms of the normal elastic force $F_{z}(P)$ :

$$
F_{x \prime}=F_{z} \frac{\sin \varphi_{P} \sqrt{1+\tan ^{2} \varphi_{P} \cos ^{2} \beta}+\mu_{0} \cos \beta}{\cos \varphi_{P} \sqrt{1+\tan ^{2} \varphi_{P} \cos ^{2} \beta}-\mu_{0} \tan \varphi_{P} \cos \beta}=F_{z} H_{x}\left(\mu_{0}, \varphi_{P}, \beta\right)
$$




$$
F_{y^{\prime}}=F_{z} \frac{\mu_{0} \sin \beta}{\cos \varphi_{P} \sqrt{1+\tan ^{2} \varphi_{P} \cos ^{2} \beta}-\mu_{0} \tan \varphi_{P} \cos \beta}=F_{z} H_{y}\left(\mu_{0}, \varphi_{P}, \beta\right)
$$

Finally, to achieve the reaction forces in the global reference system $\left(F_{x}\right.$ and $\left.F_{y}\right)$, Eq. (4) is applied, where $\alpha_{P}$ is the rotation of the local reference system in $P$ with respect to the global system, while $X$ and $Y$ are the quantities to be led back to the global reference system (i.e. the reaction forces):

$$
\left(\begin{array}{l}
X_{P} \\
Y_{P}
\end{array}\right)=\left[\begin{array}{cc}
\cos \alpha_{P} & \sin \alpha_{P} \\
-\sin \alpha_{P} & \cos \alpha_{P}
\end{array}\right]\left(\begin{array}{l}
X_{P}^{\prime} \\
Y_{P}^{\prime}
\end{array}\right)
$$

Consider now a certain number $n$ of springs, which is acting on both asperities. For each spring, the previous equations can be adopted and the total normal force $R_{z}$ will be equal to:

$$
R_{z}=\sum F_{z}^{(1)}+\sum F_{z}^{(2)}=R_{z}^{(1)}+R_{z}^{(2)}
$$

Where $R_{z}^{(1)}$ and $R_{z}^{(2)}$ are the sum of all the normal forces acting on the lower surface and on the upper surface, respectively.

The same formulations are obtained for the tangential forces in both $x$ and $y$ directions $R_{x}$ and $R_{y}$ :

$$
\begin{aligned}
& \boldsymbol{R}_{\boldsymbol{x}}^{(\mathbf{1})}=\sum \boldsymbol{F}_{x}^{(\mathbf{1})} \\
& R_{y}^{(1)}=\sum F_{y}^{(1)} \\
& R_{x}^{(2)}=\sum F_{x}^{(2)} \\
& R_{y}^{(2)}=\sum F_{y}^{(2)}
\end{aligned}
$$

From the previous relationships, in the case of no adhesion, the coefficients of friction $\mu_{x}, \mu_{y}$ and the global coefficient of friction $\mu$ are obtained as follows:

$$
\mu_{x}=\frac{R_{x}}{R_{z}} \quad \quad \mu_{y}=\frac{R_{y}}{R_{z}} \quad \mu=\frac{R_{x, y}}{R_{z}}
$$

Following the Coulomb's law, the tangential force is proportional to the normal force applied to the surface.

\subsection{Surfaces with 1D roughness}

We refer to this case when the surfaces are defined as functions of the only variable $x$ and extruded along $y$ direction, $z=f(x)$ (Figure 1.d) and thus $\varphi_{P}$ is the inverse tangent of the function $z(x)$ in $P$, reported in Figure 1.d.

Following the previous steps for the 2D case, the force equilibrium at point $\boldsymbol{P}(\boldsymbol{P} \in \boldsymbol{z}(\boldsymbol{x}))$ due to the spring interaction is obtained referring to the tangent plane $\boldsymbol{\pi}$ in $\boldsymbol{P}$ (Figure 1.e). $\beta$ is the projected direction of the sliding velocity vector on the $x y$ plane and the tangential forces $\boldsymbol{R}_{\boldsymbol{x}}$ and $\boldsymbol{R}_{\boldsymbol{y}}$ could be expressed in the following forms:

$$
\begin{aligned}
& R_{x}^{(1)}=\sum F_{z}^{(1)} H_{x}\left(\mu_{0}, \varphi_{P, 1}, \beta\right) \\
& R_{y}^{(1)}=\sum F_{z}^{(1)} H_{y}\left(\mu_{0}, \varphi_{P, 1}, \beta\right) \\
& R_{x}^{(2)}=\sum F_{z}^{(2)} H_{x}\left(\mu_{0}, \varphi_{P, 2}, \beta\right) \\
& R_{y}^{(2)}=\sum F_{z}^{(2)} H_{y}\left(\mu_{0}, \varphi_{P, 2}, \beta\right)
\end{aligned}
$$


From Eq. (10) to (13), the first part is the elastic contribution, which depends on functions $z_{1}$ and $z_{2}$, while the second belongs to one of the two asperities, to the velocity orientation $\beta$ and the local friction coefficient $\mu_{0}$.

\subsection{Surfaces with 1D wedge asperities roughness}

By adopting the surface profile as formed by tilted planes (saw tooth shape, Figure 2), the expressions of the friction forces simplify. Since the average spring displacement is equal to $\frac{\sum_{i}^{n} \Delta l_{i}}{n}$, the average normal (elastic) force on one tilted plane $\pi$ is $R_{z}^{\pi}=\frac{k_{c} \sum_{i}^{n} \Delta l_{i}}{n}=\frac{\sum_{i}^{n} k_{c} \Delta l_{i}}{n}=\frac{\sum_{i}^{n} F_{z}^{i}}{n}$ (thanks to the same spring stiffness). When the whole asperity is considered, the normal elastic force $R_{z}$ is the sum of the average elastic forces generated by the spring compression on each tilted plane, weighted by their area of competence by introducing $\psi$ and $\delta$, reported in Eq. (14). The coefficient $1 / 2$ remembers that the springs are fixed half on the upper and half on the lower surfaces.

$R_{z}=\frac{1}{2} \frac{\psi}{1+\psi} R_{z}^{(1)}+\frac{1}{2} \frac{1}{1+\psi} R_{z}^{(2)}+\frac{1}{2} \frac{\delta}{1+\delta} R_{z}^{(3)}+\frac{1}{2} \frac{1}{1+\delta} R_{z}^{(4)} \quad \boldsymbol{\psi}=\frac{\tan \varphi_{2}}{\tan \varphi_{1}} \quad \delta=\frac{\tan \varphi_{4}}{\tan \varphi_{3}}$

$R_{z}^{(1)}, R_{z}^{(2)}, R_{z}^{(3)}$ and $R_{z}^{(4)}$ are the average normal force, acting on the tilted plane by $\varphi_{1}, \varphi_{2}, \varphi_{3}$ and $\varphi_{4}$, respectively.

If $\beta=0$ the sliding motion is only along the $x$ direction, $\mu_{y}=0$ and

$\mu_{x}=\frac{\frac{1}{2} \frac{\psi}{1+\psi} R_{z}^{(1)} H_{x}\left(\mu_{0}, \varphi_{1}, \beta\right)+\frac{1}{2} \frac{\delta}{1+\delta} R_{z}^{(3)} H_{x}\left(\mu_{0}, \varphi_{3}, \beta\right)+\frac{1}{2} \frac{1}{1+\psi} R_{z}^{(2)} H_{x}\left(\mu_{0},-\varphi_{2}, \beta\right)+\frac{1}{2} \frac{1}{1+\delta} R_{z}^{(4)} H_{x}\left(\mu_{0},-\varphi_{4}, \beta\right)}{R_{z}}$

$$
=\frac{\frac{1}{2} \frac{\psi}{1+\psi} R_{z}^{(1)} \frac{\mu_{0}+\tan \varphi_{1}}{1-\mu_{0} \tan \varphi_{1}}+\frac{1}{2} \frac{\delta}{1+\delta} R_{z}^{(3)} \frac{\mu_{0}+\tan \varphi_{3}}{1-\mu_{0} \tan \varphi_{3}}+\frac{1}{2} \frac{1}{1+\psi} R_{z}^{(2)} \frac{\mu_{0}-\tan \varphi_{2}}{1+\mu_{0} \tan \varphi_{2}}+\frac{1}{2} \frac{1}{1+\delta} R_{z}^{(4)} \frac{\mu_{0}-\tan \varphi_{4}}{1+\mu_{0} \tan \varphi_{4}}}{R_{z}}
$$

On the contrary, if $\beta=90^{\circ}, \mu_{x}=0$ and

$$
\begin{gathered}
\mu_{y}=\frac{\frac{1}{2} \frac{\psi}{1+\psi} R_{z}^{(1)} H_{y}\left(\mu_{0}, \varphi_{1}, \beta\right)+\frac{1}{2} \frac{\delta}{1+\delta} R_{z}^{(3)} H_{y}\left(\mu_{0}, \varphi_{3}, \beta\right)+\frac{1}{2} \frac{1}{1+\psi} R_{z}^{(2)} H_{y}\left(\mu_{0},-\varphi_{2}, \beta\right)+\frac{1}{2} \frac{1}{1+\delta} R_{z}^{(4)} H_{y}\left(\mu_{0},-\varphi_{4}, \beta\right)}{R_{z}} \\
=\frac{\frac{1}{2} \frac{\psi}{1+\psi} R_{z}^{(1)} \frac{\mu_{0}}{\cos \varphi_{1}}+\frac{1}{2} \frac{\delta}{1+\delta} R_{z}^{(3)} \frac{\mu_{0}}{\cos \varphi_{3}}+\frac{1}{2} \frac{1}{1+\psi} R_{z}^{(2)} \frac{\mu_{0}}{\cos \varphi_{2}}+\frac{1}{2} \frac{1}{1+\delta} R_{z}^{(4)} \frac{\mu_{0}}{\cos \varphi_{4}}}{R_{z}}
\end{gathered}
$$

Eqs (15) and (16) simplify when:

- $\varphi_{1}=\varphi_{2}=\varphi, \varphi_{3}=\varphi_{4}=0$ and $\psi=\delta=1$ (Figure 2.a)

When $\beta=0, \mu_{y}=0$ and $\mu_{x}$ becomes:

$\mu_{x}=\frac{\frac{1}{4} R_{z}^{(1)} \frac{\mu_{0}+\tan \varphi}{1-\mu_{0} \tan \varphi}+\frac{1}{4} R_{z}^{(3)} \mu_{0}+\frac{1}{4} R_{z}^{(2)} \frac{\mu_{0}-\tan \varphi}{1+\mu_{0} \tan \varphi}+\frac{1}{4} R_{z}^{(4)} \mu_{0}}{R_{z}}=\frac{\mu_{0}\left(2-\sin ^{2} \varphi\left(1+\mu_{0}^{2}\right)\right)}{2\left(1-\sin ^{2} \varphi\left(1+\mu_{0}^{2}\right)\right)}$

On the contrary, if $\beta=90^{\circ}, \mu_{x}=0$ and 


$$
\mu_{y}=\frac{\frac{1}{4} R_{z}^{(1)} \frac{\mu_{0}}{\cos \varphi}+\frac{1}{4} R_{z}^{(2)} \frac{\mu_{0}}{\cos \varphi}+\frac{1}{4} R_{z}^{(3)} \mu_{0}+\frac{1}{4} R_{z}^{(4)} \mu_{0}}{R_{z}}=\frac{1}{2} \mu_{0}\left(\frac{1}{\cos \varphi}+1\right)
$$

- $\varphi_{1}=\varphi_{2}=\varphi_{3}=\varphi_{4}=\varphi$ and $\psi=\delta=1$ (Figure 2.b)

In this particular configuration, the same expressions obtained in [14] are recovered. If $\beta=0$ the sliding motion occurs in $x$ direction, $\mu_{y}=0$ and:

$$
\begin{gathered}
\mu_{x}=\frac{\frac{1}{4} R_{z}^{(1)} \frac{\mu_{0}+\tan \varphi}{1-\mu_{0} \tan \varphi}+\frac{1}{4} R_{z}^{(3)} \frac{\mu_{0}+\tan \varphi}{1-\mu_{0} \tan \varphi}+\frac{1}{4} R_{z}^{(2)} \frac{\mu_{0}-\tan \varphi}{1+\mu_{0} \tan \varphi}+\frac{1}{4} R_{z}^{(4)} \frac{\mu_{0}-\tan \varphi}{1+\mu_{0} \tan \varphi}}{R_{z}} \\
=\frac{\mu_{0}}{1-\sin ^{2} \varphi\left(1+\mu_{0}^{2}\right)}
\end{gathered}
$$

On the contrary, if $\beta=90^{\circ}, \mu_{x}=0$ and

$$
\mu_{y}=\frac{\frac{1}{4} R_{z}^{(1)} \frac{\mu_{0}}{\cos \varphi}+\frac{1}{4} R_{z}^{(3)} \frac{\mu_{0}}{\cos \varphi}+\frac{1}{4} R_{z}^{(2)} \frac{\mu_{0}}{\cos \varphi}+\frac{1}{4} R_{z}^{(4)} \frac{\mu_{0}}{\cos \varphi}}{R_{z}}=\frac{\mu_{0}}{\cos \varphi}
$$

The coefficients found in Eqs (17) and (18) are similar to the friction coefficients described by Eqs (19) and (20), but smaller. This is reasonable, because when one profile has no roughness, there is a lower contribution to enhance the global friction coefficient.

\section{Adhesion, wear and hierarchy}

\subsection{Introducing adhesion in the ALSFM}

Many times adhesion is neglected, due to the presence of surface roughness [44,45], which can remove the adhesive forces. However, even if roughness could vanish the adhesion contribution, the area of real contact is still affected by its presence [6]. In addition, adhesion must be considered if the surface is characterized by a smooth roughness and a soft material.

The most common analytical models developed to study adhesive contacts are the Johnson-Kendall-Roberts (JKR) model and the Derjaguin-Muller-Toporov (DMT) model, with related studies [46-49]. JKR model assumes an elastic sphere in contact with a flat substrate with a free energy per unit area, which occurs when the contact is reached [6,47]. Due to this energy, a contact spot with finite radius remains even if the external load is zero and this contact breaks at a critical pull-out force. The model gives the values of the contact force and the minimum contact radius at which a mechanical instability breaks the bond. If no free surface energy is present, the model collapses into the Hertz's model $[6,11,47]$. The JKR model is accurate for soft materials and high surface energy, otherwise the DMT model must be applied, which has been formulated for adhesion between hard spheres [6].

Due to a wide interest in bioinspired applications, in the last decades there has been an increase in research on adhesion [50-57]. For example, further developments of the DMT theories have shown that adhesion generates an additional load around each asperity [33]. Other works investigated the adhesion hysteresis contribution to friction [58], adhesion between surfaces with smooth roughness [45], or simulating the gecko seta in contact with rough surfaces to obtain the efficiency of the attachment $[52,56]$.

In the ALSFM, adhesion is introduced by adding the contribution of tensile springs, which can elongate to a length $l_{t}$, larger than the rest length $l_{0}$. Thus, when the applied normal load $L$ decreases, some springs can elongate when the distance between the two sliding surfaces is bigger than the spring rest length, due to the shape of a rough profile (from Figure 3.a to Figure 3.b the load $L$ is reduced). This generates a portion of the 
contact area subjected to tensile elastic forces, i.e. adhesion forces. Since the real area of contact determines the sliding friction force, adhesion, where present, can affect and modify the global frictional behaviour.

If no adhesion occurs, $R_{z}=\sum F_{z}^{(c)}$ is equal to the normal load $L$ (Figure 3.a), otherwise:

$$
L=\sum F_{z}^{(c)}+\sum F_{z}^{(t)}
$$

Where $\sum F_{z}^{(c)}>0$ is the sum of all the compressive forces and $\sum F_{z}^{(t)}<0$ is the sum of the tensile forces, which have opposite directions.

Springs are defined with different compressive and tensile behaviours, respectively $k_{c}$ and $k_{t}=k_{c} / 2$. With this assumption, the model takes into account a larger compression resistance than the tensile one. For a single spring in point $P$, the elastic force can be:

$$
\begin{gathered}
F_{Z}(P)=\left(l_{0}-l_{P}\right) k_{c}>0 \text { in case of compression; } \\
F_{z}(P)=\left(l_{0}-l_{t, P}\right) k_{t}<0 \text { in case of elongation; }
\end{gathered}
$$

It is assumed that the stretched springs do not generate a friction force by sliding, so that, the apparent coefficient of friction is expressed as follows:

$$
\mu_{a}=\frac{R_{x, y}{ }^{c}}{L}=\frac{f\left(R_{z}^{(c)}\right)}{L}
$$

Where $R_{x, y}{ }^{c}$ indicates that the tangential force $R_{x, y}$ on the $x y$ plane is generated only by the compressed springs and $f$ indicates that $R_{x, y}$ is determined only by the $R_{z}^{(c)}$ elastic forces. This implies that the apparent coefficient of friction increases due to the presence of adhesion.

\subsection{The effect of wear in the ALSFM}

Among the various tribological phenomena related to sliding friction, one of the most significant is wear. As for friction, wear involves different physical and chemical processes occurring over different time and length scales. Several empirical models have been developed, from the well-known Archard's model to recent works as experimental studies of wear track observations with Scanning Electron Microscope or Atomic Force Microscope, or even numerical simulations to obtain the surface evolution during the sliding $[6,18,33,59,60]$.

In this paper, wear is considered as a process affecting the contact surface as a function of time and is modelled following the Archard's wear law [18]:

$$
\frac{V}{S}=K \frac{L}{H}
$$

Where $V$ is the total wear (transferred) volume, $S$ is the sliding distance, $L$ is the normal load, $H$ is the surface hardness and $K$ is the wear coefficient.

It is assumed that the shape of the asperities is reduced from the initial amplitude until zero (i.e. flat) after a certain time $T_{W}$, which is the estimated time to completely smooth the surfaces with a wear rate $W=V / S$ and a constant applied load, which does not change during the wear process (Figure 4).

In the presence of $2 \mathrm{D}$ roughness, defining $S=v t$ and the wear rate $W=V / S$, the total transferred volume is:

$$
\Delta V=\iint_{x_{0}, y_{0}}^{x_{f}, y_{f}} z(x, y) d x d y-B \iint_{x_{0}, y_{0}}^{x_{f}, y_{f}} z(x, y) d x d y=W v \Delta t
$$




$$
B=1-\frac{W v \Delta t}{\iint_{x_{0}, y_{0}}^{x_{f}, y_{f}} z(x, y) d x d y}
$$

$B$ is a reduction coefficient for the roughness amplitude and varies between 0 and 1. $B=1$ means that wear has not modified the roughness yet and the transferred volume is zero. On the contrary, $B=0$ implies that the rough surfaces became flat.

When 1D roughness is considered, Eqs (24) and (25) change as follows:

$$
\begin{gathered}
\Delta V=\Delta A \cdot Y=Y \cdot\left(\int_{a}^{b} Z(x) d x-B \int_{a}^{b} Z(x) d x\right)=W v_{x} \Delta t \\
B=1-\frac{W v_{x} \Delta t}{Y \cdot \int_{a}^{b} z(x) d x}
\end{gathered}
$$

$Y$ is the asperity depth (along $y$ direction), $\Delta A$ refers to the total transferred volume per unit depth.

In the presence of $1 \mathrm{D}$ wedge asperities, wear reduces the slopes of the asperities from the initial tilt angles $\varphi^{0}$ to zero (flat surfaces, Figure 4.b). The total wear volume can also be expressed as a function of the wedge asperity angles, finding the following relationship:

$$
\frac{d \tan \varphi}{d t}=-\frac{2 W v}{s_{i}{ }^{2} Y}
$$

\subsection{Hierarchy}

When friction involves rough surfaces, the multiscale nature of their profiles can significantly affect the global coefficient of friction. For this reason, Archard proposed an hierarchical approach to describe friction in the presence of rough self-affine surfaces [61]. Hierarchical profiles and fractals have been adopted to describe also fractures in rock mechanics [62], to give an overall characterization of surface roughness $[13,29,63]$ and to model friction and contact $[13,14,30,32,64]$.

In this work, the 1D and 2D-ALSFM are extended to study the effects of rough surfaces characterized by a hierarchical structure. In this mechanical formulation, $n$ levels of hierarchy are identified, since it is assumed that each asperity presents multiple levels of roughness, one inside the other, where the upper level characteristics depend on the previous ones (Figure 5). By applying the Hierarchical ALSFM (H-ALSFM) and assuming the isotropic Coulomb friction model to be valid in every sublevel $i, \mu_{i}$ depends on its local friction coefficient $\mu_{i-1}$ as reported in Eq. (29). $\mu_{0}$ is the local coefficient of friction for the lowest 1-level, $\mu_{1}=f_{1}\left(\mu_{0}, z_{1}, z_{2}, \beta\right)$ is the global coefficient of friction at the 1-level and appears to be also the local coefficient of friction in the 2-level, and similarly for the others. By adopting a profile formed by some hierarchical self-similar levels, the surface is expressed as follows:

$$
z=A^{a} * \sin \left(\frac{2 \pi}{\lambda_{x}} B^{b} x\right) * \sin \left(\frac{2 \pi}{\lambda_{y}} C^{c} y\right)
$$

Where $A, B$ and $C$ are the coefficients that modulate the shape of the surfaces, while $a, b$ and $c$ depend on the level of roughness. In these studies it has been assumed that $A=B=C$ and $b=c=(n-i)$ while $a=-(n$ $i$ ), with $n$ number of levels, $i$ number of the actual level ( $i=1$ is for the innermost, and then it increases until $n)$.

At the $i$-level, with $\mu_{i-1}$ as the local coefficient of friction:

$$
\begin{gathered}
\mu_{i}=f_{i}\left(\mu_{i-1}, z_{1}, z_{2}, \beta\right)=\gamma\left[f_{1}\left(\mu_{0}, z_{1}, z_{2}, \beta\right)\right] \\
F_{x}=F_{z} \frac{\sin \varphi_{P} \sqrt{1+\tan ^{2} \varphi_{P} \cos ^{2} \beta}+\mu_{i-1} \cos \beta}{\cos \varphi_{P} \sqrt{1+\tan ^{2} \varphi_{P} \cos ^{2} \beta}-\mu_{i-1} \tan \varphi_{P} \cos \beta}=F_{z} H_{x}\left(\mu_{i-1}, \varphi_{P}, \beta\right)
\end{gathered}
$$




$$
\begin{gathered}
F_{y}=F_{z} \frac{\mu_{i-1} \sin \beta}{\cos \varphi_{P} \sqrt{1+\tan ^{2} \varphi_{P} \cos ^{2} \beta}-\mu_{i-1} \tan \varphi_{P} \cos \beta}=F_{z} H_{y}\left(\mu_{i-1}, \varphi_{P}, \beta\right) \\
F_{z}=K_{i} u
\end{gathered}
$$

In particular, in the presence of $1 \mathrm{D}$ wedge asperities, if $\beta=90^{\circ}$ these expressions can be simplified into:

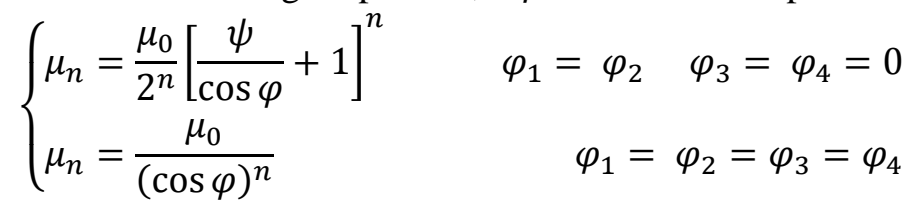

\subsection{Coupling the effect of hierarchy and wear}

In the work of Whitehouse and Archard in 1970 [17], it was found that from the profile of a rough surface after a single passage of a lubricated slider, the fine roughness (i.e. the roughness associated to the smaller levels) was removed, while the main roughness was preserved. Following the concept of such experiments, the effect of hierarchy is coupled with wear mechanisms, by assuming that the first consumed level is the innermost one. When its roughness is removed, the second begins to be subjected to wear and the coefficient of friction of the innermost level tends to its local coefficient of friction $\mu_{0}$, followed by the others until the external level is worn:

$$
\mu_{n} \rightarrow \mu_{n-1} \rightarrow \cdots \rightarrow \mu_{1} \rightarrow \mu_{0}
$$

After a time equal to $T_{w}\left(B=0, t_{0}=0\right)$, the global coefficient of friction of a certain level $i$ is reduced to its local coefficient of friction $\mu_{i-1}$ and the total number of hierarchical levels decreases from $n$ to $n-1$. In the presence of $2 \mathrm{D}$ roughness, the time needed to wear away a certain level $i$ is:

$$
T_{w, i}=\frac{\iint_{x_{0}, y_{0}}^{x_{f}, y_{f}} z(x, y) d x d y}{W v}
$$

obtained from the expression of the reduction coefficient $B$, when the latter is equal to zero.

\section{Results and Discussion}

Referring to the ALSFM, results for 1D and 2D roughness are shown in Figure 6 and Figure 7, respectively, where values of $\mu$ are normalized by $\mu_{0}$, the local coefficient of friction used in the isotropic Coulomb friction model. Six different roughness configurations are analysed by varying the sliding direction $\beta$, which strongly modifies non-linearly the coefficient of friction $\mu$. In Figure 6, generic 1D roughness (where $\varphi_{1}, \varphi_{2}$, etc., are the average slopes of the surfaces $z_{1}$ and $z_{2}$ ) has globally a higher friction coefficient than the equivalent wedge asperities. The largest coefficient of friction is reached when both the surfaces are rough and, in particular, when the first slope is greater than the following, which is the case of asymmetrical roughness (red stars in Figure 6.a-b) with respect to the rough-flat surface contacts. When $\beta$ tends to $90^{\circ}$, some reported cases tend to the same result, since for $\varphi_{1}=\varphi_{3}>\varphi_{2}=\varphi_{4}$ and $\varphi_{1}=\varphi_{3}<\varphi_{2}=\varphi_{4}$ the sliding configuration is the same when $\beta$ is $90^{\circ}$. The same occurs for $\varphi_{1}>\varphi_{2} \varphi_{3}=\varphi_{4}=0$ and $\varphi_{1}<\varphi_{2} \varphi_{3}=\varphi_{4}=0$, where the roughness asymmetry becomes negligible when the sliding is along the $y$ direction.

Referring to $2 \mathrm{D}$ roughness (Figure 7), when $z_{1}$ and $z_{2}$ are equal and symmetric, the coefficient of friction along both $x$ and $y$ directions is the same if one surface is sliding with $\beta$ equal to $0^{\circ}$ or $\beta$ equal to $90^{\circ}$, as it was expected. In addition, in this situation, the coefficient of friction does not vary as much as it does in the $1 \mathrm{D}$ case with respect to the sliding direction. This is due to the different shape of the surfaces, where the succession of summits and valleys both along $x$ and $y$ directions generates smaller frictional forces and thus the coefficient of global friction appears to be almost constant with respect to $\beta$.

More changes may occur if the wavelength of the asperities is modified (Figure 8). The coefficient of friction can increase or decrease by considering several combinations of wavelength in $x$ and $y$ directions, 
respectively $\lambda_{x}$ and $\lambda_{y}$. Globally, 25 combinations are reported, varying the sliding direction $\beta$ from $0^{\circ}$ (Figure 8.a), $45^{\circ}$ (Figure 8.c) to $90^{\circ}$ (Figure 8.b).

Figure 8. $\mathrm{a}$ and $\mathrm{b}$ are symmetrical and display the maximum coefficient of friction for the smallest wavelengths $\left(\lambda_{x}=\lambda_{y}=\pi\right)$. In addition, the bigger coefficient of friction is reached if the smaller wavelength is the one in the direction of sliding. For example, in Figure 8.a, when $\lambda_{x}=\pi$ and the direction of sliding is along the $x$ axis $(\beta=0)$ the bigger coefficient of friction is reached and appears to be slightly affected by variations in $\lambda_{y}$, while varies significantly by changing $\lambda_{x}$. Opposite results are observed in Figure 8.b, due to $\beta=90^{\circ}$.

These insights may suggest that the wavelength that mainly governs the coefficient of friction is the one in the same direction as $\beta$, because the asperities that the surfaces are facing are predominant in influencing the coefficient of friction. The increase in wavelength perpendicular to the motion slightly affects the values of $\mu$, reaching the minimum value for both the largest wavelengths. If the direction of sliding $\beta$ is equal to $45^{\circ}$, the coefficient of friction shows a symmetrical behaviour with respect to the diagonal middle values. This is reasonable because both the wavelengths play the same role in enhancing or decreasing the global coefficient of friction.

In order to validate the proposed 2D-ALSFM we compared its predictions with experimental data taken from [21], where three rough steel pins were tested against a flat ice surface. The pins profiles are modelled as reported in Figure 9, starting from the average roughness $R_{a}$ and the root mean square of the slope profiles $R_{d q}$ obtained from the profilometer. A symmetric surface morphology has been assumed in both $x$ and $y$ directions, and since the experimental sliding direction $\beta$ is random, we considered the two extreme situations found in Figure 7, i.e. $\beta=0^{\circ}$ and $\beta=30^{\circ}$. As inferred before, for $2 \mathrm{D}$ symmetric profiles the coefficient of friction is more affected by the profile morphology rather than the siding direction (circle- and diamond-shaped markers for $\beta=0^{\circ}$ and $30^{\circ}$ respectively). Furthermore, we found a good agreement between the experiments and the model predictions, where the contribution of the surface roughness is clearly shown (Figure 9.c).

When adhesion is introduced (Figure 10), several situations may occur: if all the springs are compressed (high loads), the sum of all the elastic forces balance the applied normal load $L$. Then, if the load decreases, some springs may be elongated generating tensile forces, which are balanced by the remaining compressed springs. In Figure 10.a for 1D roughness the variation in time (normalized by the period of the functions $T$ ) of tensile springs $\left(n_{t}\right)$ with respect to the total number of springs $\left(n_{t o t}\right)$ is shown, from blue to red curves. The more the load reduces, the more the springs are elongated, until $L=0$, in which the sum of the compression forces is equal in modulus to the sum of the tensile forces (opposite direction). By applying a negative load (tensile force), some springs could still be compressed (negative part of the graph in Figure 10.b).

When there is no spring elongation, the tangential force $R_{x, y}$ is linearly dependent on the normal force $R_{z}$, while if some springs start to elongate, the behaviour becomes non linear, due to adhesion effects. Moreover, adhesion appears to have a significant role only for small normal loads, as also reported in experimental works e.g. [40].

The presence of adhesion generates an (apparent) enhancement of the friction coefficient (or even a negative value, when the load has the opposite direction), as reported by Eq. (22).

When the surfaces are described by the same function, so that $z_{1}(x)=z_{2}(x)$ and the sliding direction $\beta$ is equal to $90^{\circ}$, the springs do not modify their length along the sliding. In particular, if they are in phase at $t=$ 0 , adhesion does not influence the frictional behaviour (orange curve reported in Figure 10.b).

The anisotropic roughness clearly influences the frictional response, as the curves in Figure 10.b show and the sliding direction that mainly contributes in enhancing the effects of adhesion is $\beta=0^{\circ}$.

The effect of adhesion is different if we assume a 2D roughness with sinusoidal function in both $x$ and $y$ directions (Figure 10.c-d). By displaying the tangential force $R_{x, y}$ with respect to the applied normal load $L$ it 
is evident that there is no significant dependence on the direction of sliding. Furthermore, adhesion equally affects $x$ and $y$ directions of sliding (blue line and yellow dashed line). Figure 10.d shows an enlargement on the graph close to zero. When the load $L$ is equal to zero, a frictional force is still noticeable, due to some springs that are compressed and thus generate friction.

In Figure 11 the effect of abrasive wear is introduced and discussed. As stated previously, 2D-ALSFM predictions for the global coefficient of friction are slightly affected by the direction of sliding, especially for symmetric asperities. This is clear in Figure 11.c, where the first point of each curve represents the global coefficient of friction (normalized by the local coefficient) without wear effects. These values are similar and, by adopting the same local coefficient of friction $\mu_{0}$, the results are equal to the ones reported in Figure 7. Then, when the wear mechanism starts modifying the shape of surfaces, the coefficient of friction decreases non-linearly, until it reaches the value of the local coefficient $\mu_{0}$, meaning that the surfaces became flat.

By supposing a certain wear rate $W$ (order of magnitude $10^{-3} \mathrm{~mm}^{3} / \mathrm{m}$, as reported in [59] for metals), after a time equal to $T_{W}$, the surfaces become flat and the friction coefficient tends to the local friction coefficient $\mu_{0}$. The influence of the sliding direction $\beta$ is more evident by comparing Figure 11.a-b with Figure 11.c.

Thanks to the introduction of hierarchy, the H-ASLFM firstly computes the friction coefficient of the inner level (on the smallest scale), and then the coefficient of the others. Changing a level means hypothetically changing the scale of the problem (we have assumed that the physics and the geometry of the problem are preserved). As in the previous cases, these results are obtained for distinct values of $\beta$, for both 2D and 1D roughness (wedge asperities or generic shape).

Hierarchy enhances non-linearly the friction coefficient and the coefficient of each level depends on the previous ones. By defining hierarchical sublevels in both upper and lower surfaces, the friction coefficient increases faster than in the case of only one hierarchical surface and a second single-level surface.

The effects of hierarchy in the presence of 1D sinusoidal asperities (Figure 12.a-b) and wedge asperities (Figure 12.c-d) are studied, both in the case of rough-rough or rough-flat sliding. For wedge asperities it is possible to compare the numerical results with Eqs (33) and (34). By adopting the same simplified solution in the case of sinusoidal roughness ( $\varphi$ is assumed to be the average of the slopes), it is possible to make a qualitative prediction of the variation of the friction coefficient in a hierarchical surface, even if underestimated (red curves in Figure 12.a-b), due to the slope approximation. In Figure 13 the variation of the friction coefficient between two 2D symmetric or asymmetric self-similar hierarchical asperities is reported. Three different directions of sliding $(\beta)$ are considered (Figure 13.a) and for surfaces with 2D symmetric roughness, the same behaviour is found with relative sliding along $x$ and $y$ directions, proving again that the frictional response is symmetric. However, similar results are obtained also for other $\beta$, e.g. $45^{\circ}$, meaning that the sliding direction is not the preponderant factor modifying the friction coefficient, even in the presence of hierarchical surfaces. On the contrary, if asymmetric asperities are adopted (Figure 13.b), the frictional response varies with respect to $\beta$. In particular, the results obtained for $\beta$ equal to $0^{\circ}$ or $45^{\circ}$, with surfaces characterized by $\lambda_{x}=2 \pi$ and $\lambda_{y}=3 \pi$, or by $\lambda_{x}=3 \pi$ and $\lambda_{y}=2 \pi$ show that the difference among the sliding directions is more significant, while the results for $\beta=45^{\circ}$ are the same.

When the effect of wear is introduced within the H-ASLFM, the global coefficient of friction decreases depending to the number of sublevels. In Figure 14, the coupled friction-wear behaviour between two 2D rough hierarchical surfaces is reported. $T_{W}$ is the time needed to wear away the innermost level (level 1). As stated before, each outer level is linked to the previous ones and influences the following. A change in the friction coefficient at a certain level $i$ affects all the upper levels, as it is clearly visible in Figure 14. After a time equal to $T_{W}$, the innermost level has become flat and $\mu_{n}$ reduced to $\mu_{n-1}$. 


\section{Conclusions}

We proposed a theoretical model for anisotropic dynamic friction, which considers the interactions and effects of two rough surfaces in contact, during their relative sliding. By developing the analytical model for 1D and 2D surface roughness (the Anisotropic Lattice Spring Friction Model), the parameters that influence the frictional behaviour of the model are the local friction coefficient, the direction of sliding, the amplitude and wavelength of the surfaces. The friction coefficient strongly depends on the roughness of the surfaces involved and on the sliding direction $\beta$. Generic 1D roughness displays higher friction coefficient rather than the saw tooth shape, with a reduction up to $40 \%$, moving from $\beta=0^{\circ}$ to $90^{\circ}$. By introducing $2 \mathrm{D}$ roughness, the results of this investigation highlight that symmetrical surfaces seem to be more influenced by their shape, rather than the direction of sliding, while with non-symmetrical surfaces (different wavelength in the $x$ and $y$ directions), the sliding direction affects up to 30-40\% the dynamic friction coefficient when $\beta$ varies from $0^{\circ}$ to $90^{\circ}$. We also validated the model predictions with some experimental results taken from a previous work.

Then, adhesion is introduced by imposing that the springs governing the interaction at the interface can be subjected also to tensile forces. This aspect causes an increase in both the total compression force acting on the asperities and in the apparent friction coefficient. Results show that, when adhesion affects the sliding, a tangential force is still present even if there is no normal load.

Wear is adopted as a process that reduces the friction coefficient in time and smooths the asperities. The effect is more evident if the sliding is along the profile with sharper roughness (e.g. $\beta$ equal to 0 for 1D roughness). If hierarchical self-similar levels are present, they contribute in enhancing the friction coefficient in a non-linear way. The effect of wear in the presence of hierarchical surfaces provides the model with the prediction of the friction coefficient, which decreases in time due to sliding wear.

This being the case, the present work could serve as a guide for future investigations to analyse more deeply the effects of surface topology and hierarchy in the friction response of multilevel surfaces and for the design of their tribological properties.

\section{Authors' contribution}

A.B. Conceptualization, Methodology, Software; Validation, Formal analysis, Investigation, Data curation, Writing - original draft; Writing - review and editing, Visualization.

N.M.P. Conceptualization, Methodology, Supervision, Writing - review and editing, Project administration, Funding acquisition.

\section{Funding Sources}

This work was supported by the FET Open (Boheme) grant No. 863179 as well as by Fondazione CARITRO, Cassa di Risparmio di Trento e Rovereto, No. 2019.0216.

\section{References}

[1] Dowson D. History of Tribology. London: Longman Group Limited; 1979.

[2] Popova E, Popov VL. The research works of Coulomb and Amontons and generalized laws of friction. Friction 2015;3:183-90. doi:10.1007/s40544-015-0074-6.

[3] Persson BNJ. Sliding Friction: Physical Principles and Applications. 2nd ed. Berlin, Heidelberg: Springer Berlin Heidelberg; 2000. doi:10.1007/978-3-662-04283-0.

[4] Stewart Gillmor C. Coulomb and the Evolution of Physics and Engineering in Eighteenth-Century France. Princeton: Princeton University Press; 1971.

[5] Coulomb CA. Theorie des machines simple (Theory of simple machines). Paris: Bachelier; 1821.

[6] Persson BNJ. Sliding friction: physical principles and applications. vol. 33. 2000. doi:10.1016/S01675729(98)00009-0.

[7] Caroli C, Nozières P. Hysteresis and elastic interactions of microasperities in dry friction. Eur Phys J B 1998;4:233-46. doi:10.1007/s100510050374. 
[8] Carbone G, Bottiglione F. Asperity contact theories: Do they predict linearity between contact area and load? J Mech Phys Solids 2008;56:2555-72. doi:10.1016/j.jmps.2008.03.011.

[9] Carbone G, Bottiglione F. Contact mechanics of rough surfaces: A comparison between theories. Meccanica 2011;46:557-65. doi:10.1007/s11012-010-9315-y.

[10] Capozza R, Pugno N. Effect of surface grooves on the static friction of an elastic slider. Tribol Lett 2015;58:35. doi:10.1007/s11249-015-0510-9.

[11] Hertz H. Ueber die Verdunstung der Flüssigkeiten, insbesondere des Quecksilbers, im luftleeren Raume. Ann Phys 1882;253:177-93. doi:10.1002/andp.18822531002.

[12] Greenwood JA, Williamson JBP. Contact of Nominally Flat Surfaces. Proc R Soc A Math Phys Eng Sci 1966;295:300-19. doi:10.1098/rspa.1966.0242.

[13] Majumdar a, Bhushan B. Fractal Model of Elastic-Plastic Contact between Rough Surfaces. J Tribol Asme 1991;113:1-11. doi:Doi 10.1115/1.2920588.

[14] Bhushan B, Majumdar A. Elastic-plastic contact model for bifractal surfaces. Wear 1992;153:53-64. doi:10.1016/0043-1648(92)90260-F.

[15] Persson BNJ. Elastoplastic Contact between Randomly Rough Surfaces. Phys Rev Lett 2001;87:116101. doi:10.1103/PhysRevLett.87.116101.

[16] Persson BNJ. Contact mechanics for randomly rough surfaces. Surf Sci Rep 2006;61:201-27. doi:10.1016/j.surfrep.2006.04.001.

[17] Whitehouse DJ, Archard JF. The Properties of Random Surfaces of Significance in their Contact. Proc R Soc A Math Phys Eng Sci 1970;316:97-121. doi:10.1098/rspa.1970.0068.

[18] Archard JF. Contact and rubbing of flat surfaces. J Appl Phys 1953;24:981-8. doi:10.1063/1.1721448.

[19] Caroli C, Nozières P. Hysteresis and elastic interactions of microasperities in dry friction. Eur Phys J B 1998;4:233-46. doi:10.1007/s100510050374.

[20] Mróz Z, Stupkiewicz S. An anisotropic friction and wear model. Int J Solids Struct 1994;31:1113-31. doi:10.1016/0020-7683(94)90167-8.

[21] Spagni A, Berardo A, Marchetto D, Gualtieri E, Pugno NM, Valeri S. Friction of rough surfaces on ice: Experiments and modeling. Wear 2016;368-369. doi:10.1016/j.wear.2016.10.001.

[22] Nosonovsky M, Bhushan B. Multiscale friction mechanisms and hierarchical surfaces in nano- and bio-tribology. Mater Sci Eng R Reports 2007;58:162-93. doi:10.1016/j.mser.2007.09.001.

[23] Nosonovsky M, Bhushan B. Multiscale Dissipative Mechanisms and Hierarchical Surfaces. New York: Springer Berlin Heidelberg; 2008.

[24] Berardo A, Pantano MF, Pugno NM. Slip knots and unfastening topologies enhance toughness without reducing strength of silk fibroin fibres. Interface Focus 2016;6. doi:10.1098/rsfs.2015.0060.

[25] Pantano MF, Berardo A, Pugno NM. Tightening slip knots in raw and degummed silk to increase toughness without losing strength. Sci Rep 2016;6. doi:10.1038/srep18222.

[26] Carbone G, Lorenz B, Persson BNJ, Wohlers A. Contact mechanics and rubber friction for randomly rough surfaces with anisotropic statistical properties. Eur Phys J E 2009;29:275-84. doi:10.1140/epje/i2009-10484-8.

[27] Persson BNJ, Bucher F, Chiaia B. Elastic contact between randomly rough surfaces: Comparison of theory with numerical results. Phys Rev B 2002;65:184106. doi:10.1103/PhysRevB.65.184106.

[28] Stupkiewicz S, Lewandowski MJ, Lengiewicz J. Micromechanical analysis of friction anisotropy in rough elastic contacts. Int J Solids Struct 2014;51:3931-43. doi:10.1016/j.ijsolstr.2014.07.013.

[29] Gagnepain JJ, Roques-Carmes C. Fractal approach to two-dimensional and three-dimensional surface roughness. Wear 1986;109:119-26. doi:10.1016/0043-1648(86)90257-7.

[30] Borodich FM, Onishchenko D a. Similarity and fractality in the modelling of roughness by a multilevel profile with hierarchical structure. Int J Solids Struct 1999;36:2585-612. doi:10.1016/S0020-7683(98)00116-4.

[31] Costagliola G, Bosia F, Pugno NM. Static and dynamic friction of hierarchical surfaces. Phys Rev E 2016;94:1-10. doi:10.1103/PhysRevE.94.063003.

[32] Popov VL, Dimaki A V. Using hierarchical memory to calculate friction force between fractal rough solid surface and elastomer with arbitrary linear rheological properties. Tech Phys Lett 2011;37:8-11. doi:10.1134/S106378501101010X.

[33] Vakis A, Yastrebov VA, Scheibert J, Nicola L, Dini D, Minfray C, et al. Modeling and Simulation in Tribology Across Scales: an Overview. Tribol Int 2018;125:169-99.

doi:doi.org/10.1016/j.triboint.2018.02.005. 
[34] Liskiewicz T, Morina A, Neville A. Friction in nature. WIT Trans Ecol Environ 2008;114:263-72. doi:10.2495/DN080271.

[35] Jin Z, Dowson D. Bio-friction. Friction 2013;1:100-13. doi:10.1007/s40544-013-0004-4.

[36] Pugno NM. Mimicking nacre with super-nanotubes for producing optimized super-composites. Nanotechnology 2006;17:5480-4. doi:10.1088/0957-4484/17/21/031.

[37] Pugno NM, Carpinteri A. Design of micro-nanoscale bio-inspired hierarchical materials. Philos Mag Lett 2008;88:397-405. doi:10.1080/09500830802089843.

[38] Du Y, Pugno N, Gong B, Wang D, Sun Y, Ding Q. Mechanical properties of the hierarchical honeycombs with stochastic Voronoi sub-structures. EPL (Europhysics Lett 2015;111:56007. doi:10.1209/0295-5075/111/56007.

[39] Pugno NM, Yin Q, Shi X, Capozza R. A generalization of the Coulomb's friction law: From graphene to macroscale. Meccanica 2013;48:1845-51. doi:10.1007/s11012-013-9789-5.

[40] He B, Chen W, Jane Wang Q. Surface texture effect on friction of a microtextured poly(dimethylsiloxane) (PDMS). Tribol Lett 2008;31:187-97. doi:10.1007/s11249-008-9351-0.

[41] Johnston ID, McCluskey DK, Tan CKL, Tracey MC. Mechanical characterization of bulk Sylgard 184 for microfluidics and microengineering. J Micromechanics Microengineering 2014;24. doi:10.1088/0960-1317/24/3/035017.

[42] Rand CJ, Crosby AJ. Friction of soft elastomeric wrinkled surfaces. J Appl Phys 2009;106:49131-4. doi:10.1063/1.3226074.

[43] Valentini L, Bittolo Bon S, Lopez-Manchado MA, Verdejo R, Pappalardo L, Bolognini A, et al. Synergistic effect of graphene nanoplatelets and carbon black in multifunctional EPDM nanocomposites. Compos Sci Technol 2016;128. doi:10.1016/j.compscitech.2016.03.024.

[44] Fuller KNG, Tabor D. The Effect of Surface Roughness on the Adhesion of Elastic Solids. Proc R Soc A Math Phys Eng Sci 1975;345:327-42. doi:10.1098/rspa.1975.0138.

[45] Persson BNJ, Albohr O, Tartaglino U, Volokitin AI, Tosatti E. On the nature of surface roughness with application to contact mechanics, sealing, rubber friction and adhesion. J Phys Condens Matter 2005;17:R1-62. doi:10.1088/0953-8984/17/1/R01.

[46] Derjaguin B V, Muller VM, Toporov YUP. Effect of contact deformation on the adhesion of particles. J Colloid Interface Sci 1975;52:105-8. doi:10.1016/0021-9797(75)90018-1.

[47] Johnson KL, Kendall K, Roberts AD. Surface Energy and the Contact of Elastic Solids. Proc R Soc A Math Phys Eng Sci 1971;324:301-13. doi:10.1098/rspa.1971.0141.

[48] Tabor D. Surface forces and surface interactions. J Colloid Interface Sci 1977;58:2-13. doi:10.1016/0021-9797(77)90366-6.

[49] Greenwood JA. Adhesion of elastic spheres. Proc R Soc A Math Phys Eng Sci 1997;453:1277-97. doi:10.1098/rspa.1997.0070.

[50] Autumn K, Liang YA, Hsieh ST, Zesch W, Chan WP, Kenny TW, et al. Adhesive force of a single gecko foot-hair. Nature 2000;405:681-5. doi:10.1038/35015073.

[51] Jung YC, Bhushan B. Contact angle, adhesion and friction properties of micro-and nanopatterned polymers for superhydrophobicity. Nanotechnology 2006;17:4970-80. doi:10.1088/09574484/17/19/033.

[52] Kim TW, Bhushan B. Effect of stiffness of multi-level hierarchical attachment system on adhesion enhancement. Ultramicroscopy 2007;107:902-12. doi:10.1016/j.ultramic.2006.11.008.

[53] Varenberg M, Pugno NM, Gorb SN. Spatulate structures in biological fibrillar adhesion. Soft Matter 2010;6:3269. doi:10.1039/c003207g.

[54] Pugno N, Lepore E, Toscano S, Pugno F. Normal adhesive force-displacement curves of living geckos. J Adhes 2011;87:1059-72. doi:10.1080/00218464.2011.609439.

[55] Pugno NM, Lepore E. Observation of optimal gecko's adhesion on nanorough surfaces. BioSystems 2008;94:218-22. doi:10.1016/j.biosystems.2008.06.009.

[56] Yu J, Chary S, Das S, Tamelier J, Turner KL, Israelachvili JN. Friction and adhesion of GeckoInspired PDMS flaps on rough surfaces. Langmuir 2012;28:11527-34. doi:10.1021/la301783q.

[57] Boesel LF, Cremer C, Arzt E, Campo A Del. Gecko-inspired surfaces: A path to strong and reversible dry adhesives. Adv Mater 2010;22:2125-37. doi:10.1002/adma.200903200.

[58] Carbone G, Mangialardi L. Adhesion and friction of an elastic half-space in contact with a slightly wavy rigid surface. J Mech Phys Solids 2004;52:1267-87. doi:10.1016/j.jmps.2003.12.001.

[59] Shu NP. Tribophysics. Prentice-Hall; 1986.

[60] Meng HC, Ludema KC. Wear models and predictive equations: their form and content. Wear 
1995;181-183:443-57. doi:10.1016/0043-1648(95)90158-2.

[61] Archard JF. Elastic Deformation and the Laws of Friction Article cited in : Proc R Soc Lond A 1957:190-205. doi:10.1098/rspa.1957.0214.

[62] Brown SR. A note on the description of surface roughness using fractal dimension. Geophys Res Lett 1987;14:1095-8. doi:10.1029/GL014i011p01095.

[63] Majumdar A, Tien CL. Fractal characterization and simulation of rough surfaces. Wear 1990;136:313-27. doi:10.1016/0043-1648(90)90154-3.

[64] Putignano C, Afferrante L, Carbone G, Demelio G. A new efficient numerical method for contact mechanics of rough surfaces. Int J Solids Struct 2012;49:338-43. doi:10.1016/j.ijsolstr.2011.10.009.

\section{Figures}

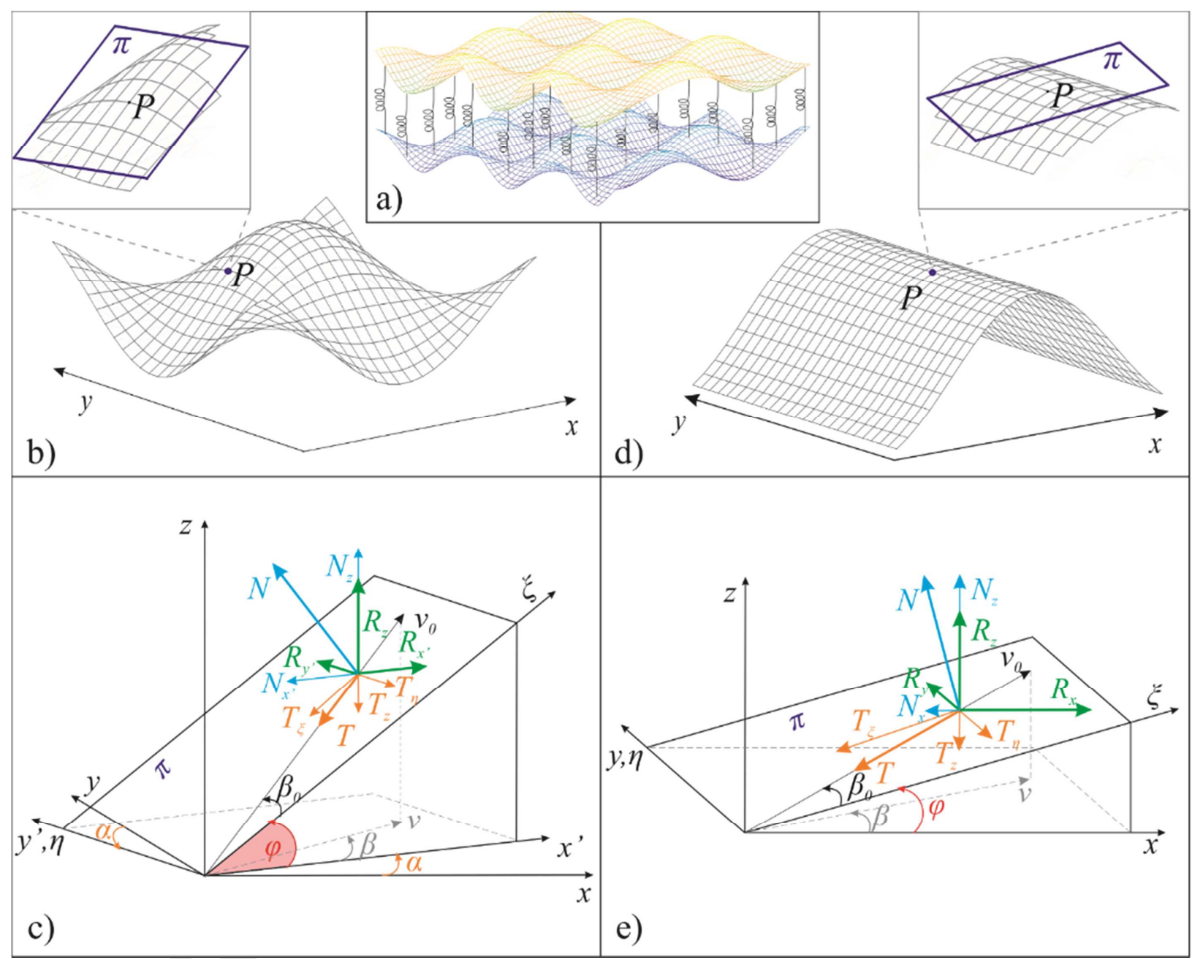

Figure 1: a) 2D roughness visualization. A set of longitudinal springs governs the interaction at the interface. b) Detail of one surface with 2D roughness: we consider the force equilibrium at point $\boldsymbol{P}(\boldsymbol{P} \in \boldsymbol{f}(\boldsymbol{x}, \boldsymbol{y}))$ due to the spring interaction. To obtain each force contribution, the tangent plane ( $\boldsymbol{\pi})$ in $\boldsymbol{P}$ is considered. c) Force decomposition on $\boldsymbol{\pi}$ with respect to the local system $\boldsymbol{x}^{\prime} \boldsymbol{y}^{\prime} \boldsymbol{z}$ in $\boldsymbol{P}$. To obtain the final reactions in the global reference system $\boldsymbol{x y z}$, the rotation matrix must be applied $(\boldsymbol{\alpha}) \boldsymbol{\beta}_{\mathbf{0}}$ identifies the direction of the sliding velocity vector $\boldsymbol{v}_{\mathbf{0}}$ on the $\boldsymbol{\pi}$ plane, tilted by $\boldsymbol{\varphi}$ from $\boldsymbol{x}^{\prime} \boldsymbol{y}^{\prime}$ plane, while $\boldsymbol{v}$ is the projected sliding velocity and $\boldsymbol{\beta}$ the projection of $\boldsymbol{\beta}_{\mathbf{0}}$ on the $\boldsymbol{x}^{\prime} \boldsymbol{y}^{\prime}$ plane. d) Detail of one surface with 1D roughness (along $\boldsymbol{x}$ direction): we consider the force equilibrium at point $\boldsymbol{P}(\boldsymbol{P} \in \boldsymbol{f}(\boldsymbol{x}))$ due to the spring interaction. To obtain each force contribution, in this case the tangent plane $(\boldsymbol{\pi})$ in $\boldsymbol{P}$ is considered as well. e) Force decomposition on $\boldsymbol{\pi}$ with respect to the local system $\boldsymbol{x y z}$ in $\boldsymbol{P}$ is considered. In this situation, no rotation matrix is needed. $\boldsymbol{\beta}_{\mathbf{0}}$ identifies the direction of the sliding velocity vector $\boldsymbol{v}_{\mathbf{0}}$ on the $\boldsymbol{\pi}$ plane, tilted by $\boldsymbol{\varphi}$ from $\boldsymbol{x} \boldsymbol{y}$ plane, while $\boldsymbol{v}$ is the projected sliding velocity and $\boldsymbol{\beta}$ the projection of $\boldsymbol{\beta}_{\mathbf{0}}$ on the $\boldsymbol{x y}$ plane.

a)

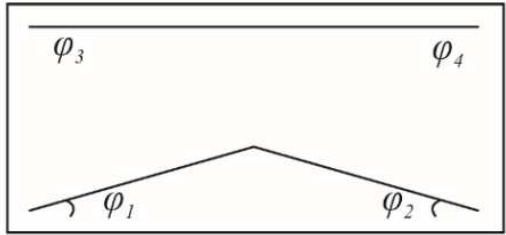

b)

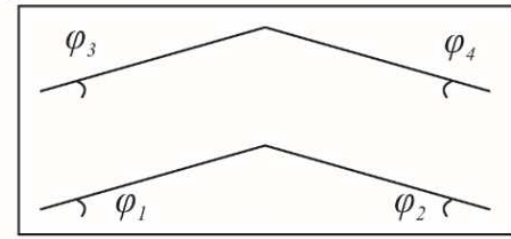

Figure 2: Particular cases of 1D wedge asperities roughness: a) One flat surface sliding on a rough surface with symmetric wedge asperities. b) Two rough surfaces with the same symmetric wedge asperities. 


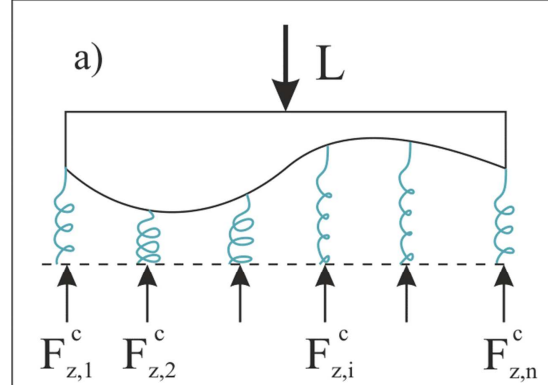

Only compression forces

c)

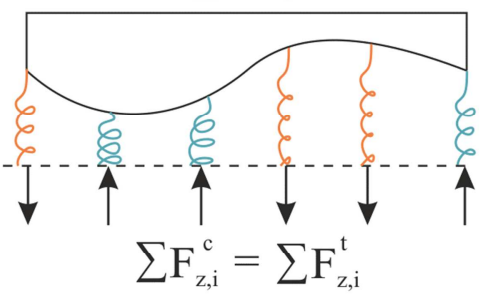

Normal load equal to zero

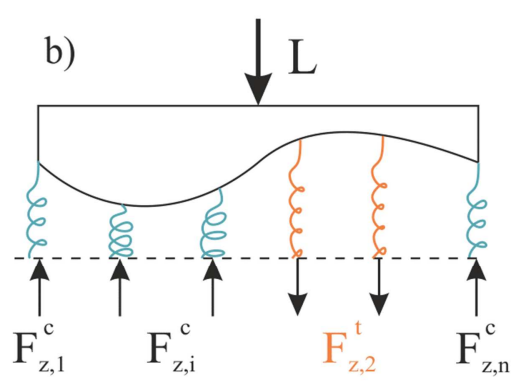

Compression/tensile forces

d)
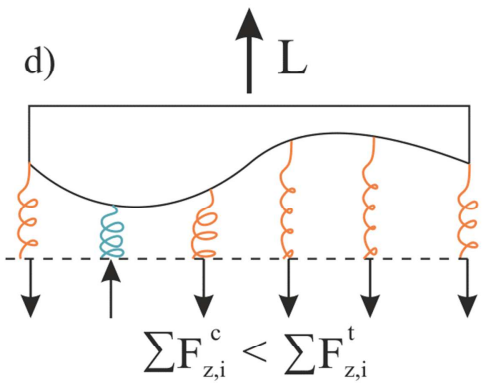

Opposite normal load

Figure 3: A 2D schematization of half model in various situations in the presence of adhesion, with decreasing $L$ from a to d. a) All the springs are compressed and the normal load is equal to the sum of all the spring axial forces. b) If some springs are elongated and $\mathrm{L}$ is the applied normal load, the sum of the compressive forces is larger than the load. c) In case of no external load, the sum of tensile and compressive forces on the springs is zero. d) In the case of a tensile load some compressed springs still generate friction.

a)

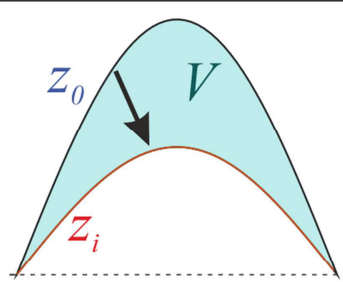

b)

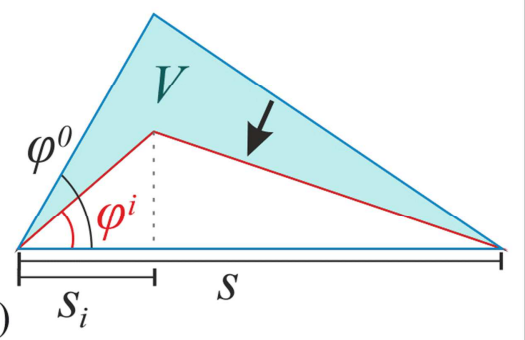

c)

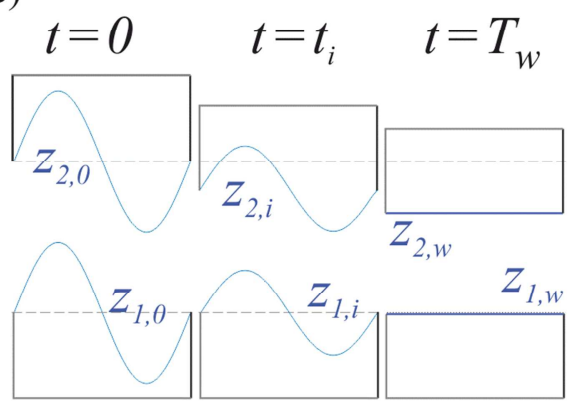

Figure 4: a) A schematization of the surface levelling after the wear process. $z_{0}$ is the initial surface shape, while $z_{i}$ is the surface profile after a certain time $t_{\mathrm{i}}$. At the end of the wear mechanism, the surface will be flat. b) A scheme of the surface levelling in case of 1D wedge asperities; $\varphi^{0}$ is the initial surface tilt angle, while $\varphi^{i}$ is the smoothed surface after a certain time $t_{i}$. c) Surface roughness changing in time. 


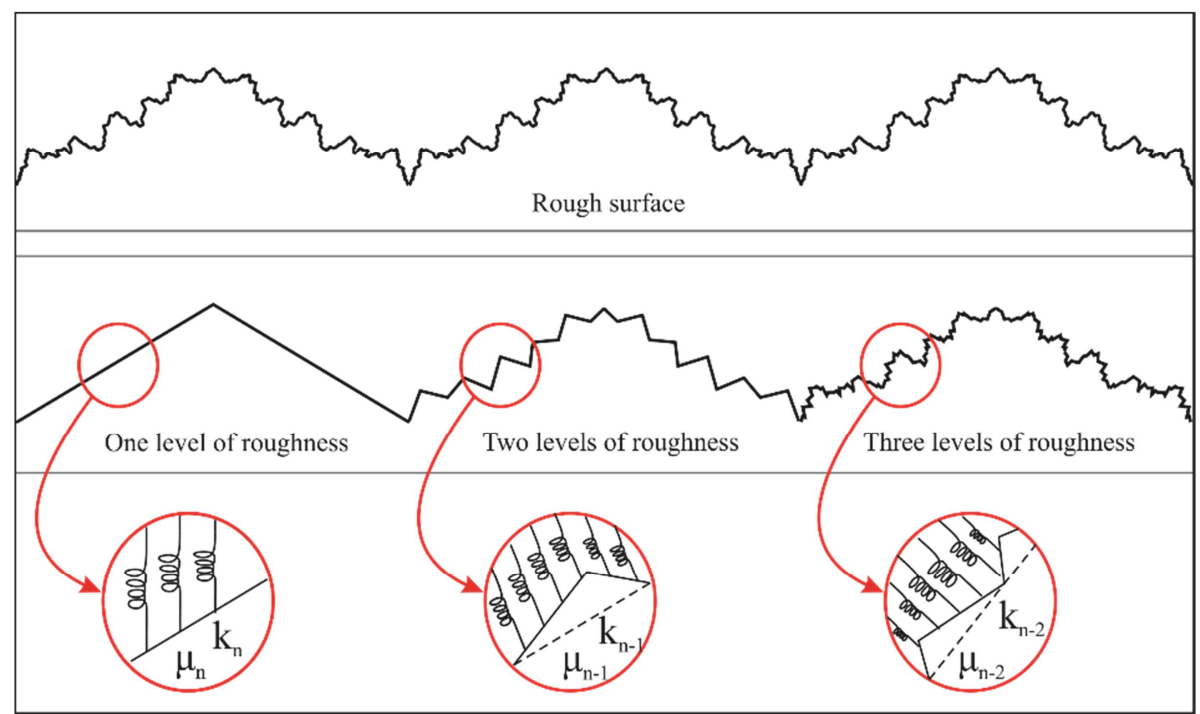

Figure 5: How to model different levels of roughness.

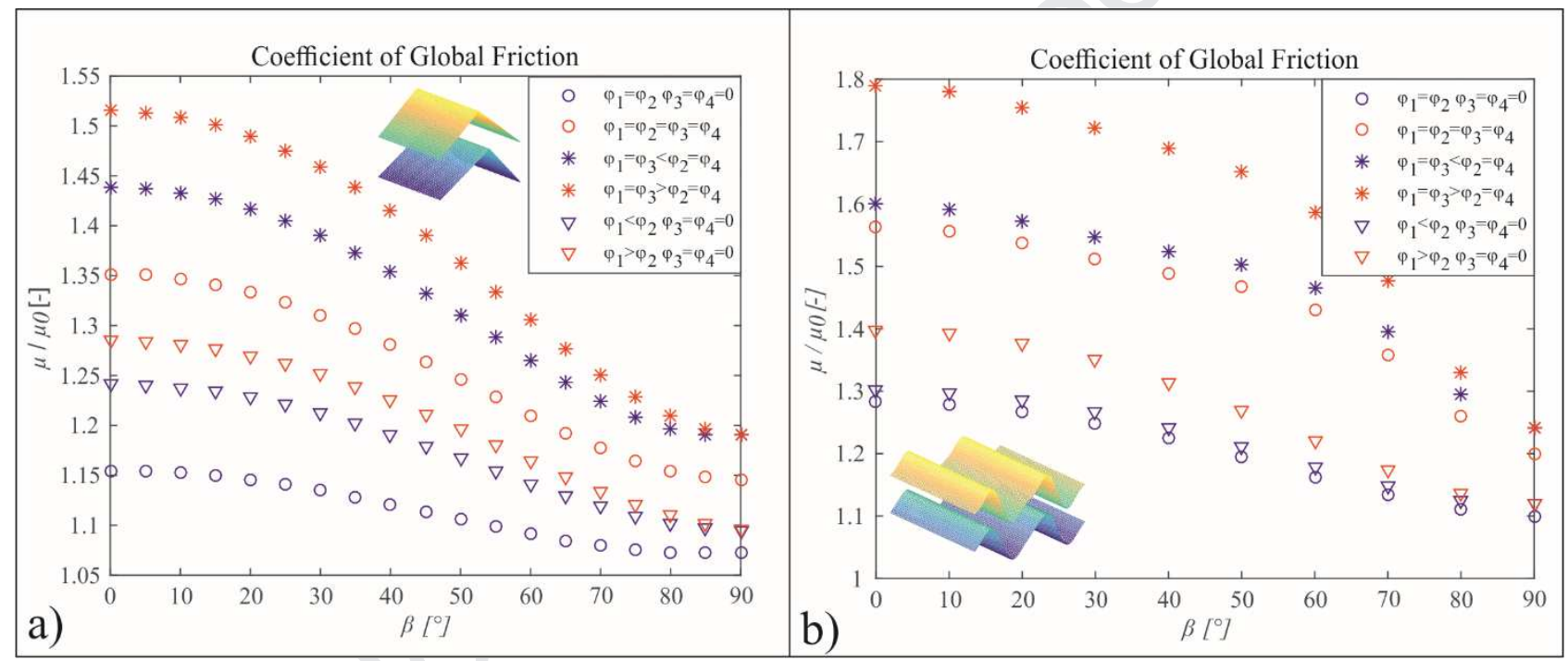

Figure 6: a) Coefficient of friction of the six case studies reported in the box in the presence of two wedge asperity surfaces. These coefficients are calculated by assuming different direction of sliding $\beta . \mathrm{b}$ ) Coefficient of friction of the six case studies reported in the box for friction between $1 \mathrm{D}$ general rough surfaces, by assuming different direction of sliding $\beta . \mu_{0}$ is set equal to 0.3 .
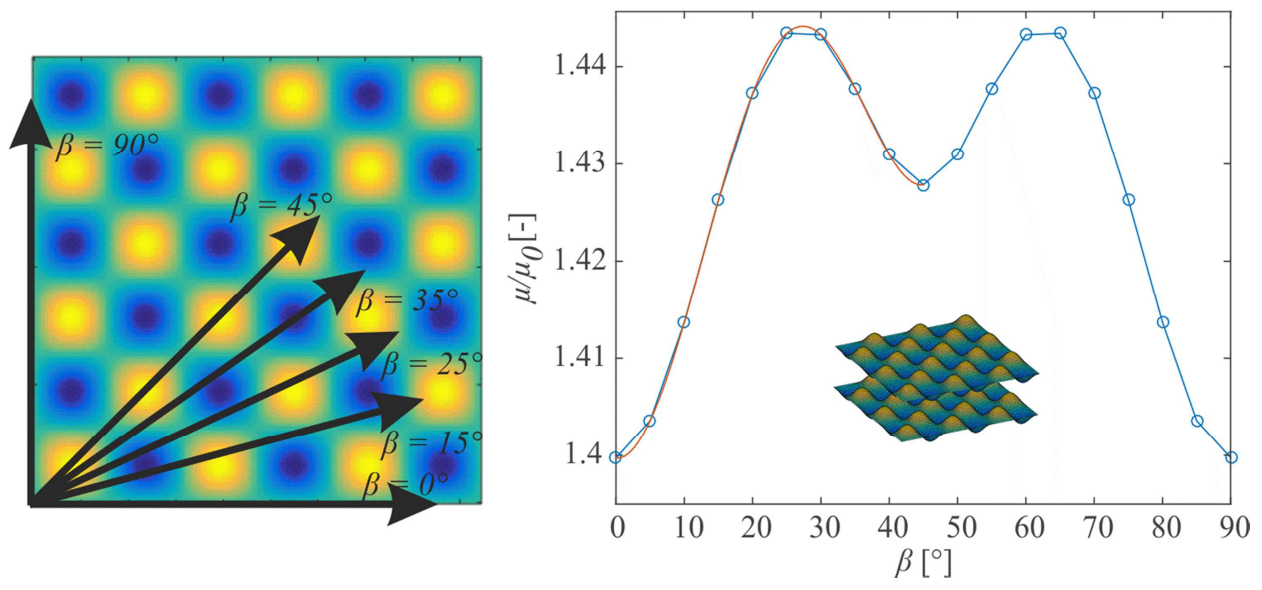

Figure 7: Variation of the coefficient of friction between two 2D rough surfaces with respect to the direction of sliding $\beta$. The upper and lower surfaces are the same, with symmetric roughness, thus the coefficient of friction $\mu$ varies between $0^{\circ}$ and $45^{\circ}$. The blue line shows how the coefficient of friction changes between $0^{\circ}$ and $90^{\circ}$, with a step $\Delta \beta$ equal to $5^{\circ}$, while the red line is the friction coefficient obtained for smaller steps. $\mu_{0}$ is set equal to 0.3 . 

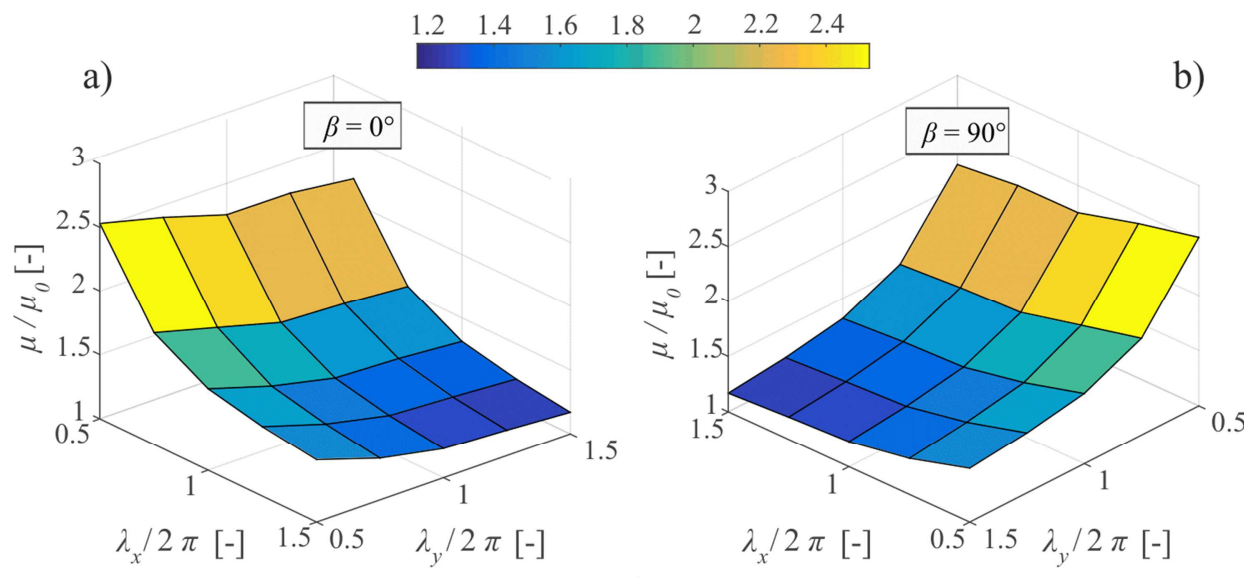

b)

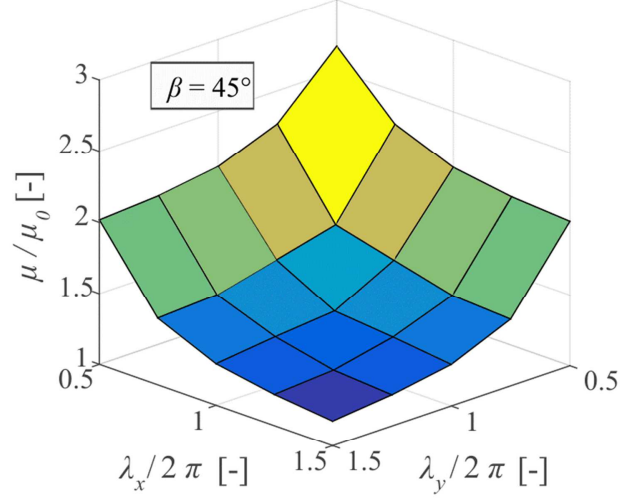

c)

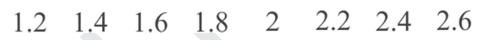

Figure 8: Changes in the friction coefficient by varying both wavelength in $x$ and $y$ directions (from $\lambda=\pi$ to $\lambda=3 \pi$ ) and sliding direction $\beta$ from $0^{\circ}$ (a) then $45^{\circ}$ (c) and finally $90^{\circ}$ (b). As expected, a and b are symmetrical and show the maximum coefficient of friction for the smallest wavelengths and this trend is preserved in the direction of sliding. Instead, the minimum value is reached for the biggest wavelengths. With $\beta$ equal to $45^{\circ}$, the coefficient of friction is symmetrical with respect to the middle values. $\mu_{0}$ is set equal to 0.3 .

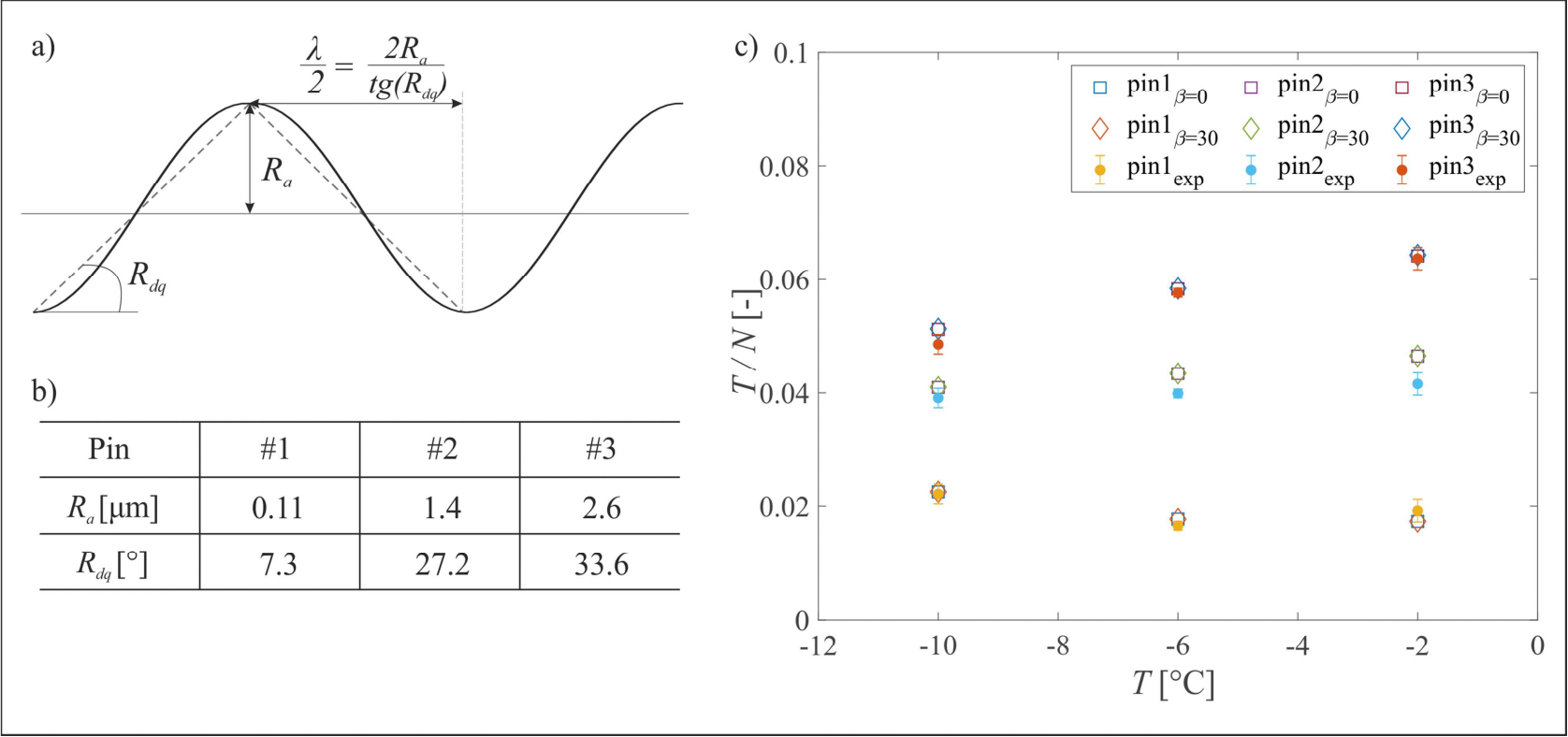

Figure 9: Comparison of the 2D model with experimental data taken from [21]. a) The pin surface are modelled assuming the average roughness $R_{a}$ and the root mean square of the slope profile $R_{d q}$ obtained from the profilometer, reported in table b). c) Comparison between the experiments (cirle-shaped markers) and the 2D-ALSFM (square-shaped and diamond-shaped markers). 


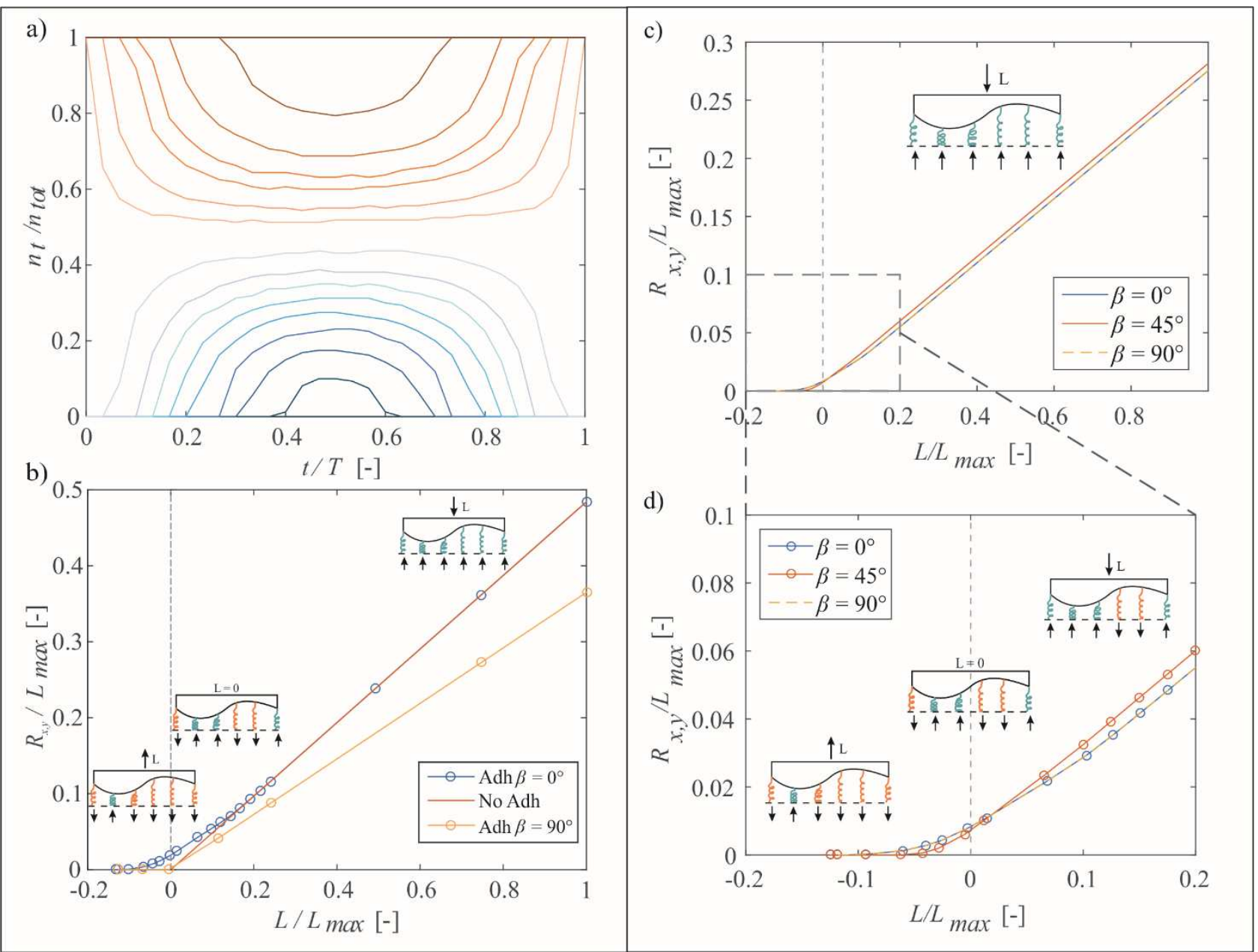

Figure 10: Effect of adhesion between 1D rough surfaces. a) Variation of springs elongation in time for 1D roughness, normalized by the function period $T$. The number of stretched springs $n_{t}$ depends on the normal load. For each circular dot in $\mathrm{b}$, a curve in a is obtained. In the reported case, until the ratio $L / L_{\max }$ is major than 0.3 , the springs are all compressed (blue line in a), corresponding to zero for each time interval). After that value, some springs are elongated, and lines from blue to red show when and how many springs display a tensile force during the sliding. When the upper and lower surfaces are both described by the same function, and they are in phase, the number of elongated springs is described by lines with shades of blue, which refer to $L>0$. On the contrary, lines with shades of red describe the number of elongated springs once the load reduces and becomes tensile $(L<0)$. b) Effect of adhesion between 1D rough surfaces. Tangential force $R_{x, y}$ with respect to the applied normal load $L$. When no adhesion occurs, the coefficient of friction is the ratio between the two; on the contrary, when some springs become tensile, a non-linear curve describes this relationship. In the presence of $1 \mathrm{D}$ roughness, adhesion does not affect the sliding with $\beta=90^{\circ}$, because there is a swift transition from compressed to tensile springs along the $y$ direction. c) Effect of adhesion between 2D rough surfaces. Tangential force $R_{x, y}$ with respect to the applied normal load $L$, both normalized with respect to the maximum load $L_{\max }$. In the presence of 2D symmetrical roughness, adhesion equally affects $x$ and $y$ directions of sliding (blue line and yellow dashed line). An enlargement on the graph close to the origin is reported in d. With other sliding directions, e.g. $\beta=45^{\circ}$, the behaviour is close to the previous cases.
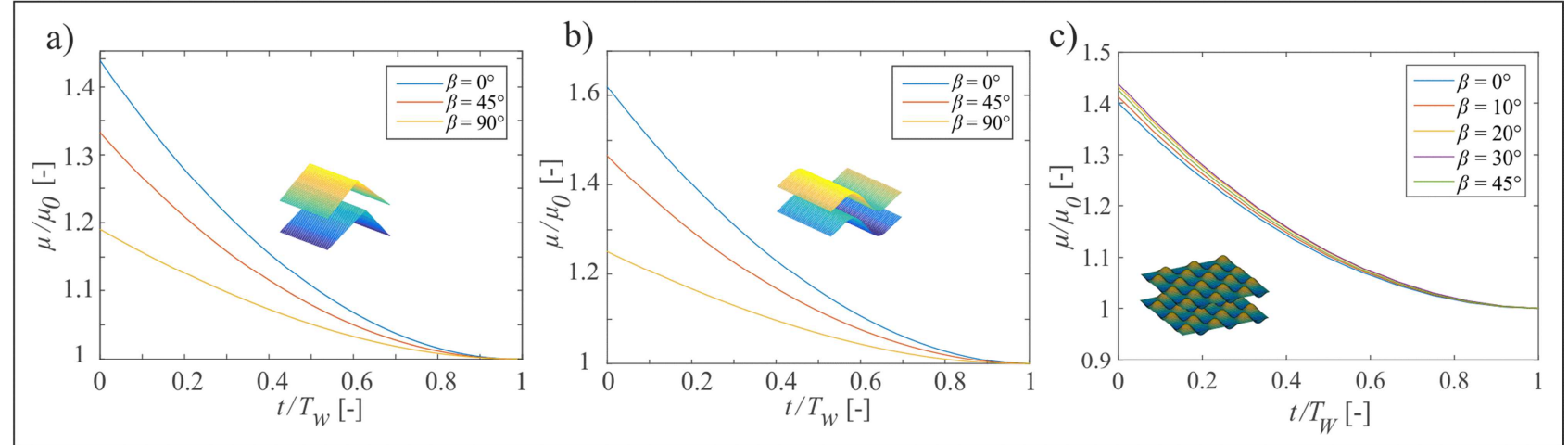

Figure 11: a) Variation in time of the friction coefficient between two wedge asperities. Three different directions of sliding $(\beta)$ have been considered. After a time equal to $T_{w}$ the surfaces become flat, thus the friction coefficient tends to the local friction 
coefficient $\mu_{0}$. b) Variation in time of the friction coefficient between two asperities with generic 1D roughness. Also here, three different directions of sliding $(\beta)$ have been considered. After a time equal to $T_{w}$ the surfaces become flat, and, as in the previous case, the friction coefficient tends to the local friction coefficient $\mu_{0}$. c) Variation in time of the friction coefficient between 2D rough symmetrical asperities. Five different directions of sliding $(\beta)$ have been considered, from $0^{\circ}$ to $45^{\circ}$ (from $45^{\circ}$ to $90^{\circ}$ the behavior is the same, due to symmetry). After a time equal to $T_{W}$ the surfaces become flat, so that the friction coefficient tends to the local friction coefficient $\mu_{0} . \mu_{0}$ is set equal to 0.3 .
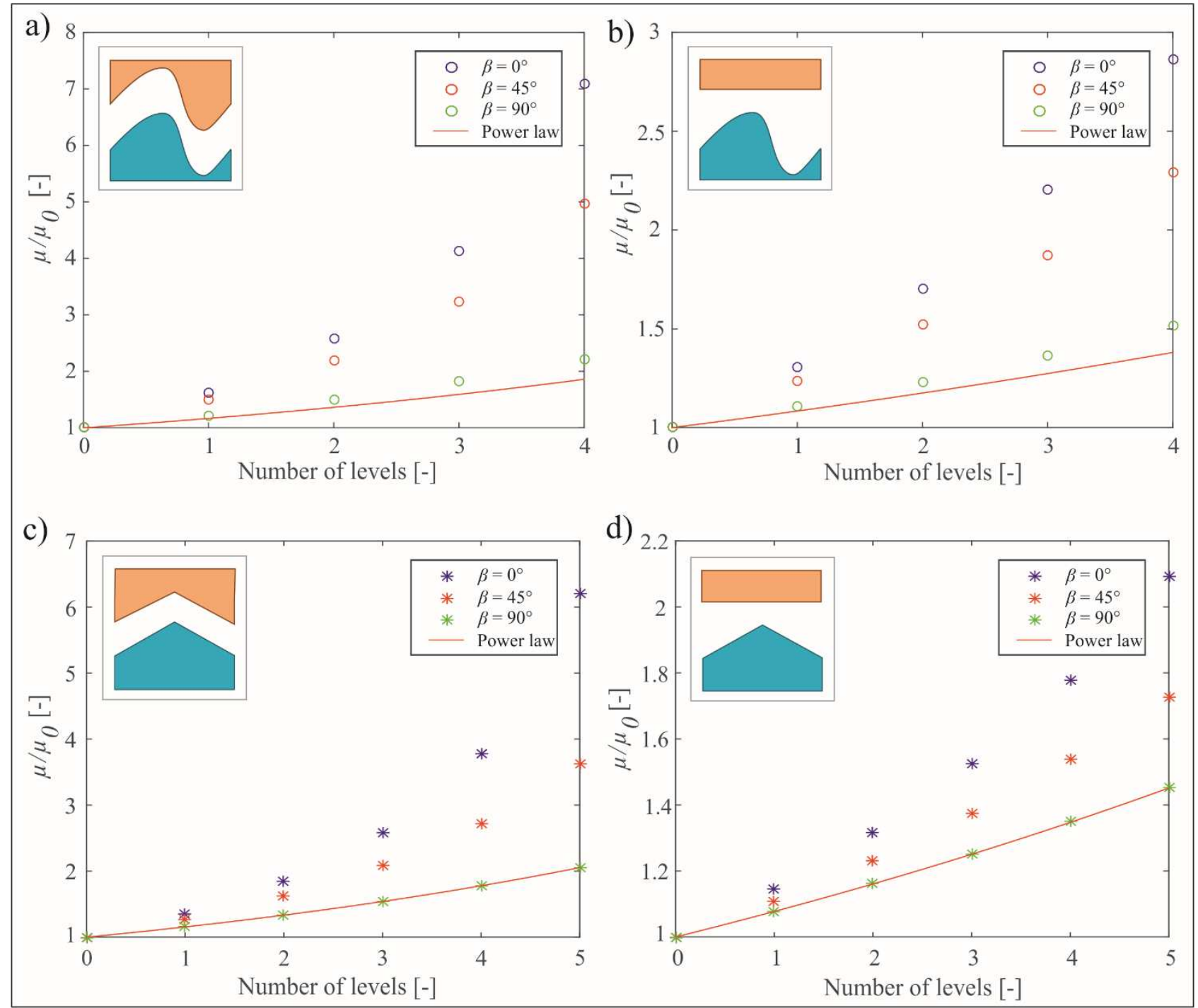

Figure 12: Hierarchical friction. The theoretical prediction, which is the exact solution in case of wedge asperities and $\beta=90^{\circ}$, is reported (red curves) for all the cases. a) Variation of the friction coefficient between two sinusoidal hierarchical asperities. Three different directions of sliding $(\beta)$ are considered. b) Variation of the friction coefficient between a sinusoidal hierarchical surface and a flat surface. Three different directions of sliding $(\beta)$ are reported. c) Variation of the friction coefficient between one hierarchical wedge asperities and a flat surface. Three directions of sliding $(\beta)$ have been reported. $d$ ) Variation of the friction coefficient between hierarchical wedge asperities. Three different directions of sliding $(\beta)$ have been considered. 


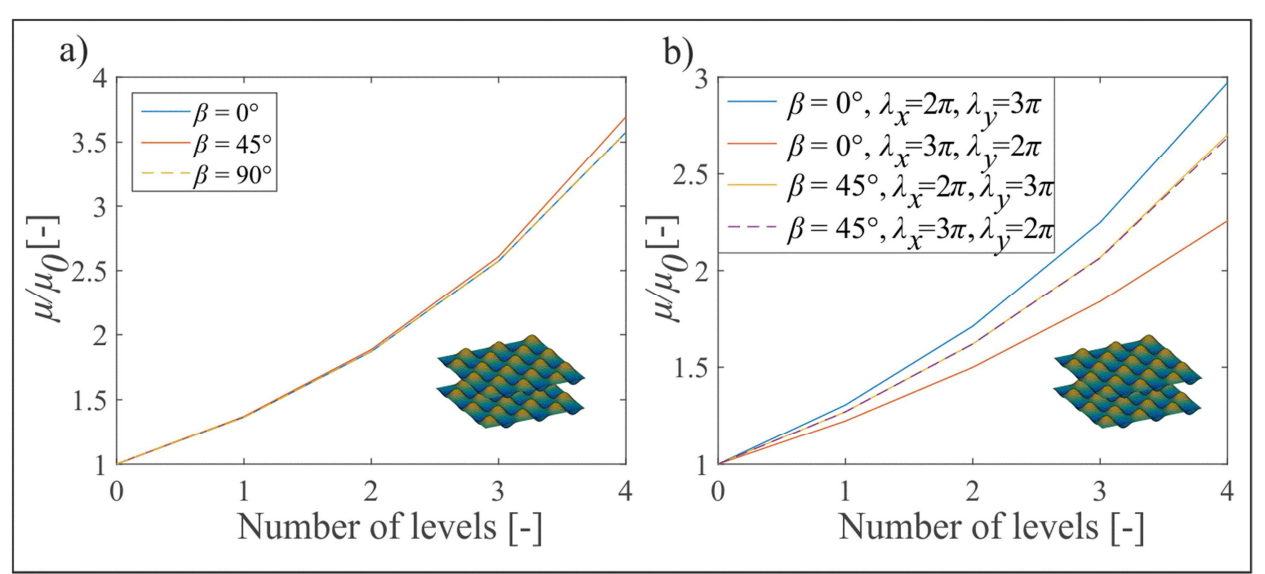

Figure 13: Hierarchical friction for 2D roughness. a) Variation of the friction coefficient between two symmetric self-similar hierarchical asperities. Three different directions of sliding $(\beta)$ are considered. b) Variation of the friction coefficient between two asymmetric self-similar hierarchical asperities. Two different directions of sliding $(\beta)$ have been considered, compared with two different asymmetric profiles. If the asperities are not symmetric, the frictional response varies in a more significant way with respect to $\beta$.

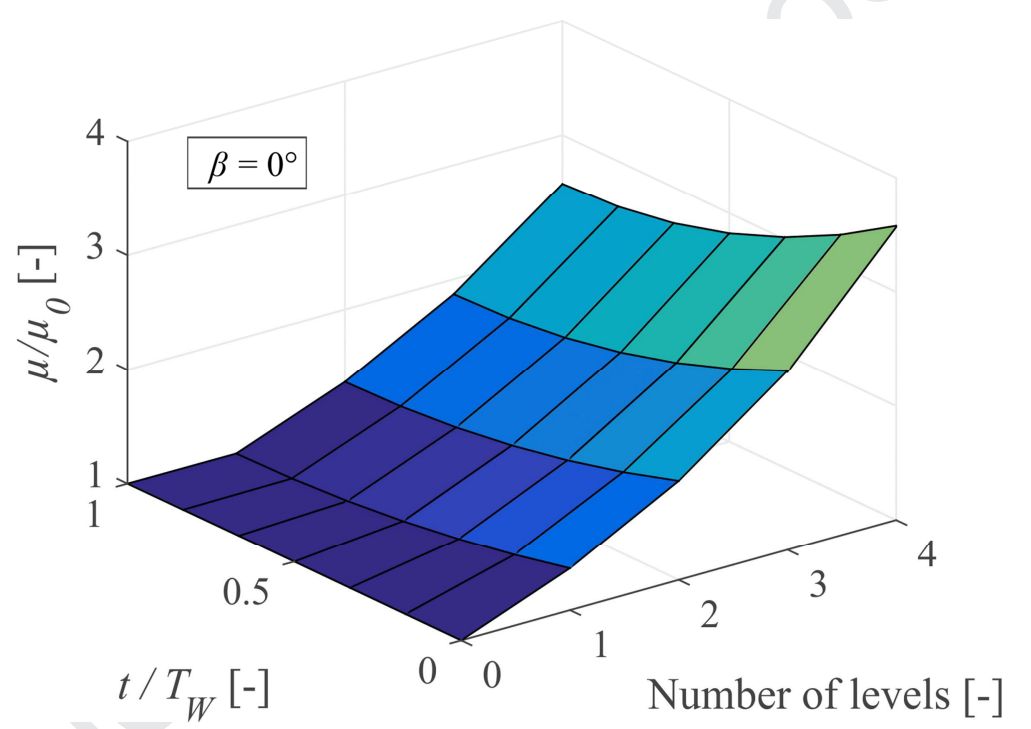

Figure 14: Variation of the global coefficient of friction as a function of wear and roughness levels for 2D roughness. Every outer level is influenced by the inner one, so that wear induces a reduction of each global coefficient of friction associated to a certain level $i$. After a time equal to $T_{W}$ the innermost level becomes flat, so that the coefficient of friction of each level at the end of this wear cycle tends to its local friction coefficient. Level 0 is the local coefficient of friction. 